\title{
Diversity of Linear Non-Ribosomal Peptide in Biocontrol Fungi
}

\author{
Xiaoyan Niu ${ }^{1}$, Narit Thaochan ${ }^{2}(\mathbb{D})$ and Qiongbo $\mathrm{Hu}^{1, *}$ \\ 1 Key Laboratory of Bio-Pesticide Innovation and Application of Guangdong Province, College of Agriculture, \\ South China Agricultural University, Guangzhou 510642, China; ny862548923@163.com \\ 2 Pest Management Biotechnology and Plant Physiology Laboratory, Faculty of Natural Resources, \\ Prince of Songkla University, Hat Yai, Songkhla 90110, Thailand; narit.t@psu.ac.th \\ * Correspondence: hqbscau@scau.edu.cn
}

Received: 13 March 2020; Accepted: 9 May 2020; Published: 12 May 2020

\begin{abstract}
Biocontrol fungi (BFs) play a key role in regulation of pest populations. BFs produce multiple non-ribosomal peptides (NRPs) and other secondary metabolites that interact with pests, plants and microorganisms. NRPs—including linear and cyclic peptides (L-NRPs and C-NRPs)—are small peptides frequently containing special amino acids and other organic acids. They are biosynthesized in fungi through non-ribosomal peptide synthases (NRPSs). Compared with C-NRPs, L-NRPs have simpler structures, with only a linear chain and biosynthesis without cyclization. BFs mainly include entomopathogenic and mycoparasitic fungi, that are used to control insect pests and phytopathogens in fields, respectively. NRPs play an important role of in the interactions of BFs with insects or phytopathogens. On the other hand, the residues of NRPs may contaminate food through BFs activities in the environment. In recent decades, C-NRPs in BFs have been thoroughly reviewed. However, L-NRPs are rarely investigated. In order to better understand the species and potential problems of L-NRPs in BFs, this review lists the L-NRPs from entomopathogenic and mycoparasitic fungi, summarizes their sources, structures, activities and biosynthesis, and details risks and utilization prospects.
\end{abstract}

Keywords: entomopathogenic fungi; mycoparasitic fungi; linear NRPs; diversity

\section{Introduction}

Biocontrol fungi (BFs) play an important role in the control of agricultural and forestry pests. BFs include mainly entomopathogenic and mycoparasitic fungi (EFs and MFs). Entomopathogenic fungi are used extensively in agricultural and medical areas. Beauveria bassiana and Metarhizium anisopliae have been developed as commercial BFs to manage many insect pests worldwide; Cordyceps spp. have been used in traditional medicines in Asia for many years [1,2]. Mycoparasitic fungi such as Trichoderma spp. have been used to control soil-borne plant diseases at commercial scales [3,4]. BFs produce multiple secondary metabolites to interact with pests, plants and microorganisms for better adapting to their environments.

Secondary metabolites produced by BFs mainly include polyketides (PKs), terpenes and non-ribosomal peptides (NRPs). NRPs are synthesized by multidomain mega-enzymes named nonribosmal peptide synthetases (NRPSs), without ribosomes and messenger RNAs. NRPSs assemble numerous NRPs with large structural and functional diversity, including more than 20 marketed drugs with antibacterial (penicillin, vancomycin), antitumor (bleomycin) and immunosuppressant (cyclosporine) activities [5]. Apart from the 20 protein amino acids, NRPs also contain rare amino acids and other organic acids. The N-terminal of NPRs are often modified by fatty acids, heterocyclic compounds, glycosylated or phosphorylated structures [6]. NRPs are divided into linear (L-NRPs) 
and cyclic NRPs (C-NRPs). Due to lack of cyclization, L-NRPs have peptide chains composed of multiple amino acids often modified by different fat chains or non-protein amino acids. L-NRPs have antimicrobial, insecticidal, antiviral or anticancer properties. There are numerous studies and reviews on fungal non-ribosomal synthases and cyclic peptides [7-9]. However, little attention has been paid to L-NRPs from BFs. As the agricultural and medical importance of BFs, it is necessary to investigate the BFs L-NRPs source species, structure, activity and biosynthesis, as well as their potential risks.

\section{L-NRPs from Entomopathogenic Fungi}

There are more than 1000 species of EFs, mainly belonging to the order Hypocreales. However, only seven L-NRPs have been reported in a few species of the genera, Cordyceps, Paecilomyces, Metarhizium and Hirsutella.

\subsection{Cicadapeptins}

Cicadapeptins are obtained from Cordyceps heteropoda ARSEF1880 [10]. There are two analogs of cicadapeptins with a modified N-terminal hydroxyproline (Hyp) acylated by n-decanoic acid and a C-terminal leucine (Leu) modified by 1,2-diamino-4-methylpentane (Figure 1). The two residues of Aib (alpha-aminoisobutyric acid) in the chain lead to the typical helical structure of cicadapeptins. Moreover, the Hyp came out twice in succession results in the structural change from helical to continuously curved one [11]. Cicadapeptins I and II show inhibitory effects on Gram-positive and negative bacteria [11].

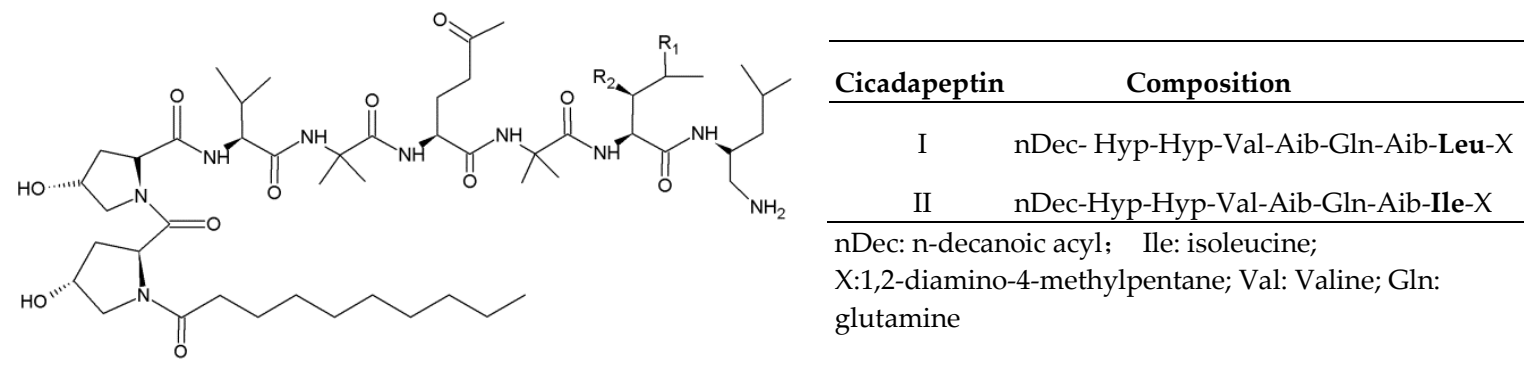

Figure 1. Basic structure and analogs of cicadapeptins

\subsection{Hirsutellic Acid A}

Hirsutellic acid A (Figure 2) is a tripeptide isolated from Hirsutella sp. BCC 1528. It has non-polar amino acids Ile, Leu and N-methyl-phenylalanine (N-Me-Phe) [12]. Hirsutellic acid A is inhibitory against Plasmodium falciparum $\mathrm{K} 1$ with an $\mathrm{IC}_{50}$ of $8 \mu \mathrm{M}$ [12].<smiles>CCC(C)[C@H](N)C(=O)N(C)[C@H](Cc1ccccc1)C(=O)N[C@@H](CC(C)C)C(=O)Nc1ccccc1C(=O)O</smiles>

Figure 2. Structural formula of hirsutellic acid A.

\subsection{Leucinostatins}

Leucinostatins can be found in Purpureocillium lilacinum (Paecilomyces lilacinus), Metarhizium marquandii (Paecilomyces marquandii) and Acremonium sp. [13-15]. About 24 analogs of leucinostatins were isolated and identified (Figure 3; Table 1). Leucinostatins have different biologic activities, 
such as antibacterial, antifungal, cytotoxic and phytotoxic properties with inhibition of oxidative phosphorylation and ATP synthesis [16]. Leucinostatins have higher activity against nematodes with $\mathrm{LD}_{50}$ of $10 \mathrm{mg} / \mathrm{L}$ [17]. Leucinostatin A inhibits the growth of prostate cancer cells by hindering insulin-like growth factor-I expression [18]. Leucinostatins also have inhibitory effects on plant parasitic oomycetes (Phytophthora spp.) [13].

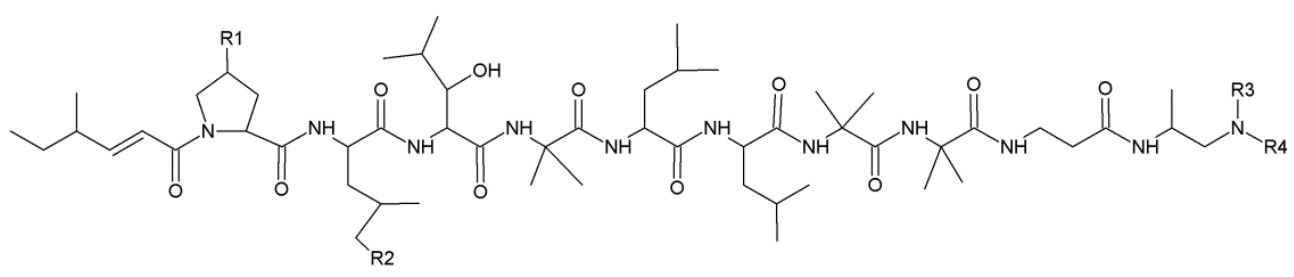

Figure 3. Basic structure of leucinostatins.

Table 1. Analogs of leucinostatins.

\begin{tabular}{|c|c|c|c|c|c|c|}
\hline Leucinostatin & Strain & $\mathbf{R}_{1}$ & $\mathbf{R}_{2}$ & $\mathbf{R}_{3}$ & $\mathbf{R}_{4}$ & Ref. \\
\hline C & P. lilacinus CG-189 & $-\mathrm{CH}_{3}$ & $-\mathrm{H}$ & $-\mathrm{H}$ & $-\mathrm{H}$ & [13] \\
\hline $\mathrm{T}$ & P. lilacinus CG-189 & $-\mathrm{H}$ & $-\mathrm{H}$ & $-\mathrm{H}$ & $-\mathrm{CH}_{3}$ & [13] \\
\hline $\mathrm{F}$ & P. lilacinus CG-189 & $-\mathrm{CH}_{3}$ & $-\mathrm{H}$ & $-\mathrm{H}$ & $-\mathrm{CH}_{3}$ & [13] \\
\hline $\mathrm{D}$ & P. lilacinus CG-189 & $-\mathrm{CH}_{3}$ & $-\mathrm{H}$ & $-\mathrm{CH}_{3}$ & $-\mathrm{CH}_{3}$ & [13] \\
\hline $\mathrm{N}$ & P. lilacinus CG-189 & $-\mathrm{CH}_{3}$ & $-\mathrm{OH}$ & $-\mathrm{H}$ & $-\mathrm{CH}_{3}$ & [13] \\
\hline $\mathrm{H}^{*}$ & $\begin{array}{l}\text { P. lilacinu CG-189 } \\
\text { P. marquandii }\end{array}$ & $-\mathrm{CH}_{3}$ & $-\mathrm{H}$ & 1 & 1 & {$[13,14]$} \\
\hline $\mathrm{B}_{2}$ & P. lilacinus CG-189 & $-\mathrm{CH}_{3}$ & $-\mathrm{CH}=\mathrm{CHCOCH}_{2} \mathrm{CH}_{3}$ & $-\mathrm{H}$ & $-\mathrm{CH}_{3}$ & [13] \\
\hline $\mathrm{V}$ & P. lilacinus CG-189 & $-\mathrm{CH}_{3}$ & $-\mathrm{CH}_{2} \mathrm{CH}_{2} \mathrm{COCH}_{2} \mathrm{CH}_{3}$ & $-\mathrm{H}$ & $-\mathrm{CH}_{3}$ & [13] \\
\hline $\mathrm{L}$ & P. lilacinus CG-189 & $-\mathrm{H}$ & $-\mathrm{CH}(\mathrm{OH}) \mathrm{CH}_{2} \mathrm{COCH}_{2} \mathrm{CH}_{3}$ & $-\mathrm{H}$ & $-\mathrm{CH}_{3}$ & [13] \\
\hline $\mathrm{A}_{2}$ & P. lilacinus CG-189 & $-\mathrm{CH}_{3}$ & $-\mathrm{CH}=\mathrm{CHCOCH}_{2} \mathrm{CH}_{3}$ & $-\mathrm{CH}_{3}$ & $-\mathrm{CH}_{3}$ & [13] \\
\hline $\mathrm{R}$ & P. lilacinus CG-189 & $-\mathrm{CH}_{3}$ & $-\mathrm{CH}_{2} \mathrm{CH}_{2} \mathrm{COCH}_{2} \mathrm{CH}_{3}$ & $-\mathrm{CH}_{3}$ & $-\mathrm{CH}_{3}$ & [13] \\
\hline B & P. lilacinus CG-189 & $-\mathrm{CH}_{3}$ & $-\mathrm{CH}(\mathrm{OH}) \mathrm{CH}_{2} \mathrm{COCH}_{2} \mathrm{CH}_{3}$ & $-\mathrm{H}$ & $-\mathrm{CH}_{3}$ & [13] \\
\hline$S$ & P. lilacinus CG-189 & $-\mathrm{CH}_{3}$ & $-\mathrm{CH}_{2} \mathrm{CH}_{2}(\mathrm{OH}) \mathrm{CHCH}_{2} \mathrm{CH}_{3}$ & $-\mathrm{CH}_{3}$ & $-\mathrm{CH}_{3}$ & [13] \\
\hline A & P. lilacinus CG-189 & $-\mathrm{CH}_{3}$ & $-\mathrm{CH}(\mathrm{OH}) \mathrm{CH}_{2} \mathrm{COCH}_{2} \mathrm{CH}_{3}$ & $-\mathrm{CH}_{3}$ & $-\mathrm{CH}_{3}$ & [13] \\
\hline U & P. lilacinus CG-189 & $-\mathrm{CH}_{3}$ & $-\mathrm{CH}\left(\mathrm{OCH}_{3}\right) \mathrm{CH}_{2} \mathrm{COCH}_{2} \mathrm{CH}_{3}$ & $-\mathrm{H}$ & $-\mathrm{CH}_{3}$ & [13] \\
\hline K & $\begin{array}{l}\text { P. lilacinus CG-189, } \\
\text { P. marquandii }\end{array}$ & $-\mathrm{CH}_{3}$ & $-\mathrm{CH}(\mathrm{OH}) \mathrm{CH}_{2} \mathrm{COCH}_{2} \mathrm{CH}_{3}$ & 1 & 1 & {$[13,14]$} \\
\hline W & P. lilacinus CG-189 & $-\mathrm{CH}_{3}$ & $-\left(\mathrm{C}_{10} \mathrm{H}_{15} \mathrm{O}_{3}\right)$ & $-\mathrm{H}$ & $-\mathrm{CH}_{3}$ & [13] \\
\hline Q & P. lilacinus CG-189 & $-\mathrm{CH}_{3}$ & $-\left(\mathrm{C}_{11} \mathrm{H}_{17} \mathrm{O}_{3}\right)$ & $-\mathrm{H}$ & $-\mathrm{CH}_{3}$ & [13] \\
\hline $\mathrm{O}$ & P. lilacinus CG-189 & $-\mathrm{CH}_{3}$ & $-\left(\mathrm{C}_{11} \mathrm{H}_{17} \mathrm{O}_{3}\right)$ & $-\mathrm{CH}_{3}$ & $-\mathrm{CH}_{3}$ & [13] \\
\hline I & P. lilacinus CG-189 & $-\mathrm{H}$ & $-\mathrm{CH}_{3}$ & $-\mathrm{CH}_{3}$ & $-\mathrm{CH}_{3}$ & [13] \\
\hline II & P. lilacinus CG-189 & $-\mathrm{H}$ & $-\mathrm{H}$ & $-\mathrm{H}$ & $-\mathrm{CH}_{3}$ & [13] \\
\hline III & P. lilacinus CG-189 & $-\mathrm{H}$ & $-\mathrm{CH}_{2} \mathrm{CH}_{2}(\mathrm{OH}) \mathrm{CHCH}_{2} \mathrm{CH}_{3}$ & $-\mathrm{CH}_{3}$ & $-\mathrm{CH}_{3}$ & [13] \\
\hline IV & P. lilacinus CG-189 & $-\mathrm{CH}_{3}$ & $-\mathrm{CH}_{2} \mathrm{CH}_{2}(\mathrm{OH}) \mathrm{CHCH}_{2} \mathrm{CH}_{3}$ & $-\mathrm{H}$ & $-\mathrm{CH}_{3}$ & [13] \\
\hline $\mathrm{V}$ & P. lilacinus CG-189 & $-\mathrm{H}$ & $-\mathrm{CH}(\mathrm{OH}) \mathrm{CH}_{2} \mathrm{COCH}_{2} \mathrm{CH}_{3}$ & $-\mathrm{CH}_{3}$ & $-\mathrm{CH}_{3}$ & [13] \\
\hline
\end{tabular}

* The C- terminal $-\mathrm{N}\left(\mathrm{R}_{3}\right) \mathrm{R}_{4}$ of leucinostatin $\mathrm{H}$ is altered with $-\mathrm{CH}_{2} \mathrm{CH}_{2} \mathrm{ONH}_{2}$.

\subsection{Efrapeptins}

Efrapeptins are mainly produced by Tolypocladium niveum (Tolypocladium inflatum, Beauveria nivea) and Tolypocladium geodes [19-21] but are also found in Acremonium sp. and Metarhizium anisopliae [20,22]. Efrapeptins have 10 analogs with molecular weight of 1592-1676 Da [22]. The analogs of efrapeptins contain alpha-azide carboxylic acid (Figure 4, Table 2). Efrapeptins have antifungal, antimalarial, insecticidal and antitumor activities [20,23]. In particular, they can inhibit the ATPase and, disturb the interaction between ATPase and heat shock protein 90 (Hsp90) [24-26]. Actually, not only the F1-ATPase, but also the vacuolar ATPase (V-ATPase) in brush border membrane vesicles (BBMV) can be inhibited [27]. Efrapeptin J inhibit gastric cancer cells HT1080 [28]. 
<smiles>[R]NC(CC(C)C)C[N+]1=C2CCCN2CCC1</smiles>

Figure 4. Basic structure and analogs of efrapeptins.

Table 2. The analogs of efrapeptins.

\begin{tabular}{|c|c|c|c|}
\hline Efrapeptin & Strain & $\mathbf{R}$ & Ref. \\
\hline $\mathrm{A}$ & T. niveum ARSEF NO.616 & Ac-Aib-Gly-Leu-Iva- & [20] \\
\hline $\mathrm{B}$ & T. niveum ARSEF NO.616 & Ac-Leu-Iva- & [20] \\
\hline $\mathrm{C}$ & $\begin{array}{l}\text { T. niveum ARSEF NO.616, } \\
\text { T. geodes ARSEF } 2684\end{array}$ & $\begin{array}{l}\text { Ac-Pip-Aib-Pip-Aib-Aib-Leu- } \beta \text { Ala-Gly-Aib- } \\
\text { Aib-Pip-Aib-Gly-Leu-Aib- }\end{array}$ & {$[19,20]$} \\
\hline $\mathrm{D}$ & $\begin{array}{l}\text { T. niveum ARSEF } 616 \text {, } \\
\text { T. geodes ARSEF 2684, } \\
\text { M. anisopliae ME1 }\end{array}$ & $\begin{array}{l}\text { Ac-Pip-Aib-Pip-Aib-Aib-Leu- } \beta \text { Ala-Gly-Aib- } \\
\text { Aib-Pip-Aib-Gly-Leu-Iva- }\end{array}$ & {$[19,20]$} \\
\hline $\mathrm{E}$ & $\begin{array}{l}\text { T. niveum ARSEF } 616 \text {, } \\
\text { T. geodes ARSEF } 2684\end{array}$ & $\begin{array}{l}\text { Ac-Pip-Aib-Pip-Iva-Aib-Leu- } \beta \text { Ala-Gly-Aib- } \\
\text { Aib-Pip-Aib-Gly-Leu-Iva- }\end{array}$ & {$[19,20]$} \\
\hline $\mathrm{E}_{\alpha}$ & Acremonium sp. 021172c & $\begin{array}{l}\text { Ac-Pip-Aib-Pip-Iva-Aib-Leu- } \beta \text { Ala-Gly-Aib- } \\
\text { Aib-Pip-Aib-Ala-Leu-Aib- }\end{array}$ & [22] \\
\hline $\mathrm{F}$ & $\begin{array}{l}\text { T. niveum ARSEF } 616 \text {, } \\
\text { T. geodes ARSEF 2684, } \\
\text { M. anisopliae ME1 }\end{array}$ & $\begin{array}{l}\text { Ac-Pip-Aib-Pip-Aib-Aib-Leu- } \beta \text { Ala-Gly-Aib- } \\
\text { Aib-Pip-Aib-Ala-Leu-Iva- }\end{array}$ & {$[19,20]$} \\
\hline G & $\begin{array}{l}\text { T. niveum ARSEF } 616 \text {, } \\
\text { T. geodes ARSEF } 2684\end{array}$ & $\begin{array}{l}\text { Ac-Pip-Aib-Pip-Iva-Aib-Leu- } \beta \text { Ala-Gly-Aib- } \\
\text { Aib-Pip-Aib-Ala-Leu-Iva- }\end{array}$ & {$[19,20]$} \\
\hline $\mathrm{H}$ & $\begin{array}{l}\text { T. geodes ARSEF 2684, } \\
\text { Acremonium sp. 021172c }\end{array}$ & $\begin{array}{l}\text { Ac-Pip-Aib-Pip-Iva-Iva-Leu- } \beta \text { Ala-Gly-Aib- } \\
\text { Aib-Pip-Aib-Ala-Leu-Iva- }\end{array}$ & {$[19,22]$} \\
\hline $\mathrm{J}$ & Tolypocladium sp. AMB18 & $\begin{array}{l}\text { Ac-Pip-Aib-Pip-Aib-Aib-Leu- } \beta \text { Ala-Gly-Aib- } \\
\text { Aib-Pip-Aib-Ala-Leu-Aib- }\end{array}$ & [28] \\
\hline
\end{tabular}

Ac: acetyl; Pip: 4-aminopiperidine-4-carboxyl; Iva: S-isovaline; $\beta$ Ala: $\beta$-Alanine; Gly: glycine.

\subsection{Peptaibol Compounds}

Peptaibols are a special kind of L-NRPs which have been found in a variety of soil fungi. Most of them are found in Trichoderma spp. which have been used to control plant disease [29]. To date, more than 500 peptaibols have been identified [30] and among them, 35 types were identified after 2000 [31]. Peptaibols are rich in non-protein amino acid and are often acetylated at the N-terminus and hydroxylated at the C-terminus. Peptaibols contain 5-20 aa residues forming $\alpha$-helical conformation [32,33]. Moreover, peptaibols form ion channels on the lipid bilayer membrane. Those peptaibols with long sequences (12-20 aa) have the "barrel-stave" ion channel, while, the others with short sequences (5-11 aa) possess the "carpet" ion channel as a dimers with their N-termini connected (Figure 5) [34]. Therefore, peptaibols can break the ion balance of cells leading to functional disorder of cell. They not only have antibacterial, cytotoxic activity, but also are teratogenic to the larvae of some marine organisms [35,36]. In BFs, there are three peptaibols reported.

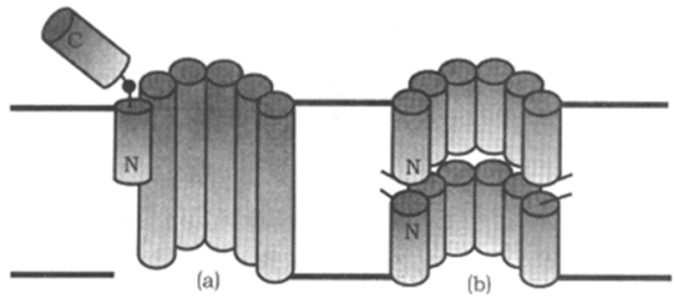

Figure 5. Peptaibols act as ion channels in membrane [34]. (a) "barrel-stave" ion channel model; (b) "carpet" ion channel model. 


\subsubsection{Culicinins}

The entomopathogenic fungus Culicinomyces clavisporus LL-12I252 [37,38] produces culicinins. They are decapeptides with four analogs (A-D) with the sketch of Bta-Pro-Ahmod-Aib/AlaAib/Ala-Amd-Leu-Aib-Leu-Apa-Apae-OH (Figure 6). Culicinin D can selectively inhibit MDA 468 breast cancer cells [38].

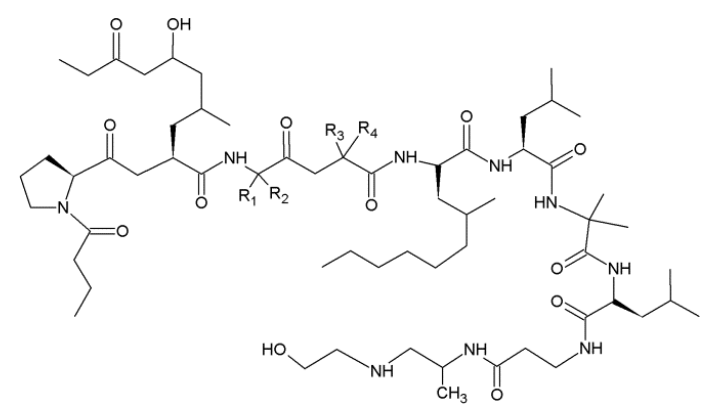

\begin{tabular}{cl}
\hline Culicinin & \multicolumn{1}{c}{$\mathbf{R}$} \\
\hline A & $\mathrm{R} 1=\mathrm{R} 3=\mathrm{H}, \mathrm{R} 2=\mathrm{R} 4=\mathrm{Me}$ \\
$\mathrm{B}$ & $\mathrm{R} 1=\mathrm{R} 2=\mathrm{R} 3=\mathrm{Me}, \mathrm{R} 4=\mathrm{H}$ \\
$\mathrm{C}$ & $\mathrm{R} 1=\mathrm{H}, \mathrm{R} 2=\mathrm{R} 3=\mathrm{R} 4=\mathrm{Me}$ \\
$\mathrm{D}$ & $\mathrm{R} 1=\mathrm{R} 2=\mathrm{R} 3=\mathrm{R} 4=\mathrm{Me}$ \\
\hline
\end{tabular}

Bta: butyric acid; Apa: 3-aminopropionic acid;

Ahmod: 2-amino-6-hydroxy-4-methyl-8-oxydecanoic acid; Amd: 2-amino-4-methyl-dodecanoic acid;

Apae-OH : 2-(2'-aminopropyl) aminoethanol.

Figure 6. Basic structure and analogs of culicinins.

\subsubsection{LP237}

The entomopathogenic fungus Tolypocladium geodes (Beauveria geodes) produces LP237 with three analogs (Table 3) [39-41]. The highly helical structure of LP237 and the amphiphilic side chain of amino acids form a "barrel-stave" ion channel on the membrane, the Gln 6 , Gln 7 and Gln 10 in the peptide are on the same polar surface of the helix, forming a cavity of the ion channel, resulting in the membrane permeability activity of the peptide [42]. LP237 F8 is cytotoxic to P388D1 mouse leukemia cells and human tumor cells, such as lung cancer A549, ovarian cancer OVCAR3, colon cancer SW620 and breast cancer MCF7. It has synergistic actions with other anticancer peptides [42].

Table 3. Analogs of LP237.

\begin{tabular}{cc}
\hline LP237 & Composition \\
\hline F8 & nOca-Aib-Pro-Phe-Aib-Gln-Gln-Aib-Et-Nva-Gln-Ala-Leuol \\
F5 & nOca-Aib-Pro-Tyr-Aib-Gln-Gln-Aib-Et-Nva-Gln-Ala-Leuol \\
F7 & nDec-Aib-Pro-Phe-Aib-Gln-Gln-Aib-Aib-Gln-Ala-Leuol \\
\hline \multicolumn{2}{c}{ Leuol: Leucinol; Et-Nva: 2-amino-3-ethylpentanoic acid; Tyr: Tyrosine. }
\end{tabular}

\subsubsection{Metanicins}

Metanicins are produced by strain CBS 597.80 of Metarhizium. There are four analogs with 20 aa residues and are similar to the peptaibols (longibrachin, trichobrachins, trichokonins) from Trichoderma (Table 4). Metanicins inhibit bacteria with the activity order of Micrococcus lutes $>$ Enterococcus faecalis $>$ Staphylococcus aureus $>$ Bacillus subtilis [43].

Table 4. Analogs of metanicins.

\begin{tabular}{cl}
\hline Metanicins & Composition \\
\hline A & Ac-Aib-Ala-Aib-Ala-Aib-Ala-Gln-Aib-Val-Aib-Gly-Leu-Aib-Pro-Val-Aib-Aib-Gln-Gln-Pheol \\
B & Ac-Aib-Ala-Aib-Ala-Aib-Ala-Gln-Aib-Val-Aib-Gly-Leu-Aib-Pro-Val-Aib-D-Iva-Gln-Gln- Pheol \\
C & Ac-Aib-Ala-Aib-Ala-Aib-Aib-Gln-Aib-Val-Aib-Gly-Leu-Aib-Pro-Val-Aib-Aib-Gln-Gln-Pheol \\
D & Ac-Aib-Ala-Aib-Ala-Aib-Aib-Gln-Aib-Val-Aib-Gly-Leu-Aib-Pro-Val-Aib-D-Iva-Gln-Gln-Pheol \\
\hline
\end{tabular}




\section{L-NRPs from Mycoparasitic Fungi}

There were 14 L-NRPs found in mycoparasitic fungi mainly in the genus of Trichoderma and Penicillium. Apart from $\delta$-(L- $\alpha$-aminoadipyl)-L-cysteinyl-D-valine (ACV), the other $13 \mathrm{~L}-\mathrm{NRPs}$ all belong to peptaibols.

\section{1. $A C V$}

$\mathrm{ACV}$ is a tripeptide formed by condensation of L-aminoadipic acid, L-cysteine and L-valine (Figure 7). It is a synthetic precursor of the antibiotics penicillin and cephalosporins [44]. ACV was isolated from Penicillium chrysogenum, Cephalosporins acremonium and Aspergillus nidulans. Interestingly, Penicillium chrysogenum is the most important ACV producer, it not only increases plant resistance to pathogens [45], but also has insecticidal activity to Bactrocera oleae [46]. ACV is synthesized by ACV synthase (ACVS) which was from fungi and bacteria [47-49].<smiles>CC(C)C(NC(=O)C(CS)NC(=O)CCCC(N)C(=O)O)C(=O)O</smiles>

Figure 7. Structural formula of $\delta$-(L- $\alpha$-aminoadipyl)-L-cysteinyl-D-valine.

\subsection{Harzianins}

Harzianins are named because first, identified in extract of Trichoderma harzianum. T. harzianum not only has a good inhibitory effect on plant pathogens, but also be used to control mosquito pests [50]. To date, there are up to 15 harzianin analogs (Table 5). The HC type contains three kinks formed by the Aib-Pro motifs. The structures are 310 -helices, which are embedded in the lipid layer to form a voltage-gated ion channel of the "barrel-stave" type, which increases the hydrophobicity and permeability of the lipid bilayer [51,52]. The PCU4 type are similar to HC, but with shorter chain [53]. Compared to HC type, the HB I is missing an Aib-Pro-Ala [54]. There are two Aib-Pro motifs in the HK VI, also in the $3_{10}$-helices conformation [55]. The HA V only contains an Aib-Pro, forming the center hinge of Pro's $\alpha$-helix structure [56].

Table 5. Analogs of harzianins.

\begin{tabular}{|c|c|c|c|c|c|}
\hline Harzianin & Residue & Analogs & Strain & Composition & Ref. \\
\hline $\mathrm{HC}$ & 14 & 11 & $\begin{array}{l}\text { T. harzianum } \\
\text { M-903603 }\end{array}$ & $\begin{array}{l}\text { Ac-Aib-Gln/Asn-Leu-Aib-Pro-Ala/Ser-Ile/Val- } \\
\text { Aib-Pro-Iva/Aib-Leu-Aib-Pro-Leuol }\end{array}$ & [51] \\
\hline HB I & 11 & 1 & $\begin{array}{l}\text { T. harzianum } \\
\text { M-903603 }\end{array}$ & $\begin{array}{l}\text { Ac-Aib-Asn-Leu-Ile-Aib-Pro-Iva-Leu-Aib- } \\
\text { Pro-Leuol }\end{array}$ & [54] \\
\hline HK VI & 11 & 1 & Trichoderma sp. & $\begin{array}{l}\text { Ac-Aib-Asn-Ile-Ile-Aib-Pro-Leu-Leu-Aib- } \\
\text { Pro-Leuol }\end{array}$ & [55] \\
\hline HA V & 18 & 1 & $\begin{array}{l}\text { T. harzianum } \\
\text { M-903603 }\end{array}$ & $\begin{array}{l}\text { Ac-Aib-Gly-Ala-Aib-Iva-Gln-Aib-Val-Aib- } \\
\text { Gly-Leu-Aib-Pro-Leu-Aib-Iva-Gln-Leuol }\end{array}$ & [56] \\
\hline PCU4 & 14 & 1 & T. harzianum & $\begin{array}{l}\text { Ac-Aib-Asn-Leu-Aib-Pro-Ser-Ile-Aib-Pro- } \\
\text { Aib-Leu-Aib-Pro-Valinol }\end{array}$ & [53] \\
\hline
\end{tabular}

\subsection{Trichorzins}

Trichorzins are 18 aa peptaibols with up to 10 analogs found in T. harzianum and T. virens (Table 6). Trichorzin PAs with six analogs found in T. harzianum show the higher activity against mycoplasma and spiroplasma [57,58]. Three TVB analogs were isolated from T. virens [59]. Trichorzins PAs have a polar C-terminus of tryptophan (Trpol) with affinity to the hydrophilic head of the phospholipid molecule in bilayer membrane, which is important for construction of a voltage-gated ion channel of these "barrel-stave" peptaibols [60]. 
Table 6. Analogs of trichorzins.

\begin{tabular}{|c|c|c|c|}
\hline Trichorzin & Strain & Composition & Ref. \\
\hline PA II & T. harzianum M-902608 & $\begin{array}{l}\text { Ac-Aib-Ser-Ala-Aib-Iva-Gln-Aib-Val-Aib-Gly-Leu- } \\
\text { Aib-Pro-Leu-Aib-Aib-Gln-Trpol }\end{array}$ & [57] \\
\hline PA IV & T. harzianum M-902608 & $\begin{array}{l}\text { Ac-Aib-Ser-Ala-Aib-Iva-Gln-Iva-Val-Aib-Gly-Leu- } \\
\text { Aib-Pro-Leu-Aib-Aib-Gln-Trpol }\end{array}$ & [57] \\
\hline PA V & T. harzianum M-902608 & $\begin{array}{l}\text { Ac-Aib-Ser-Ala-Iva-Iva-Gln-Aib-Val-Aib-Gly-Leu- } \\
\text { Aib-Pro-Leu-Aib-Aib-Gln-Trpol }\end{array}$ & [57] \\
\hline PA VI & T. harzianum M-902608 & $\begin{array}{l}\text { Ac-Aib-Ser-Ala-Aib-Iva-Gln-Aib-Val-Aib-Gly-Leu- } \\
\text { Aib-Pro-Leu-Aib-Aib-Gln-Pheol }\end{array}$ & [57] \\
\hline PA VIII & T. Harzianum M-902608) & $\begin{array}{l}\text { Ac-Aib-Ser-Ala-Aib-Iva-Gln-Iva-Val-Aib-Gly-Leu- } \\
\text { Aib-Pro-Leu-Aib-Aib-Gln-Pheol }\end{array}$ & [57] \\
\hline PA IX & T. harzianum M-902608 & $\begin{array}{l}\text { Ac-Aib-Ser-Ala-Iva-Iva-Gln-Aib-Val-Aib-Gly-Leu- } \\
\text { Aib-Pro-Leu-Aib-Aib-Gln-Pheol }\end{array}$ & [57] \\
\hline PAU4 & T. harzianum M-902608 & $\begin{array}{l}\text { Ac-Aib-Ser-Ala-Aib-Aib-Gln-Aib-Val-Aib-Gly-Leu- } \\
\text { Aib-Pro-Leu-Aib-Aib-Gln-Trpol }\end{array}$ & [57] \\
\hline TVB I & T. virens TV29-8 & $\begin{array}{l}\text { Ac-Aib-Gly-Ala-Val-Aib-Gln-Aib-Ala-Aib-Ser-Leu- } \\
\text { Aib-Pro-Leu-Aib-Aib-Gln-Valol }\end{array}$ & [59] \\
\hline TVB II & T. virens TV29-8 & $\begin{array}{l}\text { Ac-Aib-Gly-Ala-Leu-Aib-Gln-Aib-Ala-Aib-Ser-Leu- } \\
\text { Aib-Pro-Leu-Aib-Aib-Gln-Valol }\end{array}$ & [59] \\
\hline TVB IV & T. virens TV29-8 & $\begin{array}{l}\text { Ac-Aib-Gly-Ala-Leu-Aib-Gln-Iva-Ala-Aib-Ser-Leu- } \\
\text { Aib-Pro-Leu-Aib-Aib-Gln-Valol }\end{array}$ & [59] \\
\hline
\end{tabular}

Ser: serine.

\subsection{Longibrachins}

Trichoderma longbrachitum has strong inhibition against the soil-borne phytopathogens, Rhizoctonia solani, Sclerotium rolfsii and Pythium aphanidermatum [61]. In addition, it also has insecticidal and nematocidal activity, respectively against insect Leucinodes orbonalis and Heterodera avenae [62,63].

T. longibrachiatum produces longibrachin (LG), which is a peptaibol with 20 aa residues. Six LG analogs were found. The A series LGs with four analogs (LG A I-IV) have the neutral Gln at 18th aa residues, while the B series (LG B II-III) are replaced with acidic Glu (Table 7) [64,65]. The negatively charged side chain Glu of LG B increases the oligomerization level of the ion channel and improves the transportation of substances [66]. LGs result in deformities of Crassostrea gigas larvae and may be neurotoxic to Calliphora vomitoria with an $\mathrm{ED}_{50}$ of $270 \mathrm{mg} / \mathrm{kg}[64,67]$. They are also toxic effect on KB cells (human oral epidermoid cancer cells) [64]. LGs show antibacterial activity against mycoplasma and Gram-positive bacteria. LG AIV also shows a weak inhibition of human pathogenic fungus Aspergillus fumigatus [64,65].

Table 7. Analogs of longibrachins.

\begin{tabular}{|c|c|c|c|}
\hline Longibrachin & Strain & Composition & Ref. \\
\hline LG A I & T. longbrachitum MMS151 & $\begin{array}{l}\text { Ac-Aib-Ala-Aib-Ala-Aib-Ala-Gln-Aib-Val-Aib- } \\
\text { Gly-Leu-Aib-Pro-Val-Aib-Aib-Gln } 18 \text {-Gln-Pheol }\end{array}$ & [64] \\
\hline LG A II & T. longbrachitum MMS151 & $\begin{array}{l}\text { Ac-Aib-Ala-Aib-Ala-Aib-Ala-Gln-Aib-Val-Aib- } \\
\text { Gly-Leu-Aib-Pro-Val-Aib-Iva-Gln } 18 \text {-Gln-Pheol }\end{array}$ & [64] \\
\hline LG A III & T. longbrachitum MMS151 & $\begin{array}{l}\text { Ac-Aib-Ala-Aib-Ala-Aib-Aib-Gln-Aib-Val-Aib- } \\
\text { Gly-Leu-Aib-Pro-Val-Aib-Aib-Gln } 1{ }_{18} \text {-Gln-Pheol }\end{array}$ & [64] \\
\hline LG A IV & T. longbrachitum MMS151 & $\begin{array}{l}\text { Ac-Aib-Ala-Aib-Ala-Aib-Aib-Gln-Aib-Val-Aib- } \\
\text { Gly-Leu-Aib-Pro-Val-Aib-Iva-Gln } 18 \text {-Gln-Pheol }\end{array}$ & [64] \\
\hline LG B II & $\begin{array}{l}\text { T. longbrachitum } \\
\text { LCP-853431 }\end{array}$ & $\begin{array}{l}\text { Ac-Aib-Ala-Aib-Ala-Aib-Ala-Gln-Aib-Val-Aib- } \\
\text { Gly-Leu-Aib-Pro-Val-Aib-Aib-Glu }{ }_{18} \text {-Gln-Pheol }\end{array}$ & [65] \\
\hline LG B III & $\begin{array}{l}\text { T. longbrachitum } \\
\text { LCP-853431 }\end{array}$ & $\begin{array}{l}\text { Ac-Aib-Ala-Aib-Ala-Aib-Ala-Gln-Aib-Val-Aib- } \\
\text { Gly-Leu-Aib-Pro-Val-Aib-Iva-Glu } 18 \text {-Gln-Pheol }\end{array}$ & [65] \\
\hline
\end{tabular}

\subsection{Trichobrachins}

Trichobrachins are purified from T. longibrachiatum and T. parceramosum [68]. There are 11 analogs (Table 8) [68,69]. Trichobrachins inhibit Bacillus subtilis [68]. 
Table 8. Analogs of trichobrachins.

\begin{tabular}{clll}
\hline Trichobrachin & Strain & Composition & Ref. \\
\hline A I & T. longibrachiatum & Ac-Aib-Asn-Leu-Leu-Aib-Pro-Leu-Aib-Aib-Pro-Leuol & {$[69]$} \\
A II & T. longibrachiatum & Ac-Aib-Asn-Leu-Leu-Aib-Pro-Val-Leu-Aib-Pro-Valol & {$[69]$} \\
A III & T. longibrachiatum & Ac-Aib-Asn-Val-Leu-Aib-Pro-Leu-Leu-Aib-Pro-Valol & {$[69]$} \\
A IV & T. longibrachiatum & Ac-Aib-Asn-Leu-Val-Aib-Pro-Leu-Leu-Aib-Pro-Valol & {$[69]$} \\
B I & T. longibrachiatum & Ac-Aib-Asn-Leu-Leu-Aib-Pro-Val-Aib-Val-Pro-Leuol & {$[69]$} \\
B II & T. longibrachiatum & Ac-Aib-Asn-Val-Leu-Aib-Pro-Leu-Aib-Val-Pro-Leuol & {$[69]$} \\
B III & T. longibrachiatum & Ac-Aib-Asn-Leu-Val-Aib-Pro-Leu-Aib-Val-Pro-Leuol & {$[69]$} \\
B IV & T. longibrachiatum & Ac-Aib-Asn-Leu-Leu-Aib-Pro-Leu-Aib-Val-Pro-Valol & {$[69]$} \\
I & T. parceramosum CBS & Ac-Aib-Ala-Ala/Aib-Ala-Aib-Ala/Aib-Gln-Aib-Vxx- & {$[68]$} \\
& 936.69 & Aib-Gly-Leu-Aib-Pro-Vxx-Aib-Aib/Vxx-Gln-Gln & \\
& & Ac-Aib-Ala-Ala/Aib-Ala-Aib-Ala/Aib-Gln-Aib-Vxx- & [68] \\
II & T. parceramosum CBS & Aib-Gly-Lxx-Aib-Pro-Vxx-Aib-Aib/Vxx/Ala-Gln & \\
& 936.69 & Ac-Aib-Ala-Ala/Aib-Ala-Aib-Ala/Aib-Gln-Aib-Vxx-Aib- & [68] \\
& T. parceramosum CBS & Gly-Lxx-Aib-Pro-Vxx-Aib-Aib/Vxx/Ala-Gln-Gln-Pheol & Ac-Aib-Asn/Gln-Vxx/Lxx- \\
III & 936.69 & Vxx/Lxx-Aib-Pro-Lxx-Vxx/Lxx-Aib-Pro-Lxxol/Valol & \\
\end{tabular}

Vxx: Val/Isovaline; Lxx: Leu/Ile; Lxxol: Leucinol/isoleucinol.

\subsection{Trichogins}

Trichogin GA IV is purified from T. longibrachiatum strain M3431. It has a sequence of nOct-Aib-Gly-Leu-Aib-Gly-Gly-Leu-Aib-Gly-Ile- Leuol (Figure 8) [70,71]. Trichogin has a N-terminus of n-octanoic acid and contains four hydrophobic glycine, which lead to a hybrid structure with the $3_{10}$-helix and the $\alpha$-helix of trichogen $[72,73]$. Trichogin has low hemolytic activity and obvious antibacterial activity against methicillin-resistant Staphylococcus aureus [72,74]. Trichogin is also toxic to several human tumor cells (mononuclear HL60 in myelogenous leukemia, HeLa in ovarian sarcoma, A431 in epidermoid cancer and A549 in lung cancer) $[73,75]$.

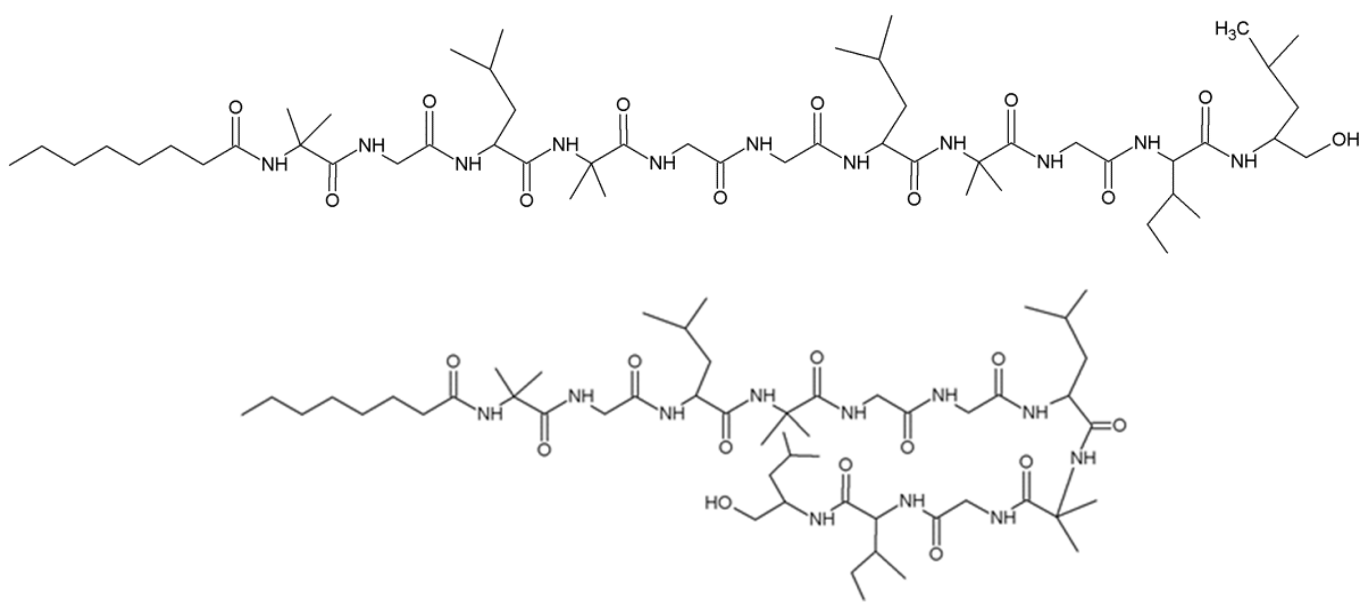

Figure 8. Structure of trichogin GA IV.

\subsection{Trilongins}

Trilongins have 13 analogs with 11 or 20aa residues and are mainly found in T. longibrachiatum and T. atroviride (Table 9) [76,77]. The trilongin A series have 11aa residues with average molecular weight of $1175 \mathrm{Da}$, while the trilongin B and C series have 20aa residues with 1936-1965 Da. Trilongins are toxic to mammals. They destroy the mitochondria of boar sperm cells, remarkably, the mixtures of long and short sequences trilongins are more toxic [77]. Trilongins form voltage-gated $\mathrm{K}^{+} / \mathrm{Na}^{+}$ion channels, moreover, the combinations of A type with $\mathrm{B} / \mathrm{C}$ type than the single type have synergistic effect to keep the ion channel open longer [77]. 
Table 9. Analogs of trilongins

\begin{tabular}{|c|c|c|c|}
\hline Trilongin & Strain & Composition & Ref. \\
\hline AIV a & T. longibrachiatum & Ac-Aib-Asn-Vxx-Vxx-Aib-Pro-Vxx-Lxx-Aib-Pro-Lxxol & [77] \\
\hline $\mathrm{AIV} b$ & T. longibrachiatum & Ac-Aib-Asn-Vxx-Vxx-Aib-Pro-Lxx-Lxx-Aib-Pro-Vxxol & [77] \\
\hline AIV c & T. longibrachiatum & Ac-Aib-Asn-Vxx-Vxx-Aib-Pro-Lxx-Vxx-Aib-Pro-Lxxol & [77] \\
\hline AIII a & T. longibrachiatum & Ac-Aib-Asn-Lxx-Vxx-Aib-Pro-Lxx-Lxx-Aib-Pro-Vxxol & [77] \\
\hline AIII b & T. longibrachiatum & Ac-Aib-Asn-Lxx-Vxx-Aib-Pro-Vxx-Lxx-Aib-Pro-Lxxol & [77] \\
\hline AIII c & T. longibrachiatum & Ac-Aib-Asn-Vxx-Lxx-Aib-Pro-Lxx-Lxx-Aib-Pro-Vxxol & [77] \\
\hline AIII d & T. longibrachiatum & Ac-Aib-Asn-Vxx-Lxx-Aib-Pro-Vxx-Lxx-Aib-Pro-Lxxol & [77] \\
\hline AII a & T. longibrachiatum & Ac-Aib-Asn-Lxx-Lxx-Aib-Pro-Lxx-Lxx-Aib-Pro-Vxxol & [77] \\
\hline AII b & T. longibrachiatum & Ac-Aib-Asn-Lxx-Lxx-Aib-Pro-Lxx-Vxx-Aib-Pro-Lxxol & [77] \\
\hline AII c & T. longibrachiatum & Ac-Aib-Asn-Lxx-Lxx-Aib-Pro-Vxx-Lxx-Aib-Pro-Lxxol & [77] \\
\hline AII d & T. longibrachiatum & Ac-Aib-Asn-Lxx-Vxx-Aib-Pro-Lxx-Lxx-Aib-Pro-Lxxol & [77] \\
\hline AII e & T. longibrachiatum & Ac-Aib-Asn-Vxx-Lxx-Aib-Pro-Lxx-Lxx-Aib-Pro-Lxxol & [77] \\
\hline AI & T. longibrachiatum & Ac-Aib-Asn-Lxx-Lxx-Aib-Pro-Lxx-Lxx-Aib-Pro-Lxxol & [77] \\
\hline A0 & T. longibrachiatum & Ac-Aib-Gln-Lxx-Lxx-Aib-Pro-Lxx-Lxx-Aib-Pro-Lxxol & [77] \\
\hline BI & T. longibrachiatum & $\begin{array}{l}\text { Ac-Aib-Ala-Aib-Ala-Aib-Ala-Gln-Aib-Vxx-Aib-Gly-Lxx- } \\
\text { Aib-Pro-Vxx-Aib-Aib-Gln-Gln-Pheol }\end{array}$ & [77] \\
\hline BII & T. longibrachiatum & $\begin{array}{l}\text { Ac-Aib-Ala-Aib-Ala-Aib-Ala-Gln-Aib-Vxx-Aib-Gly-Lxx- } \\
\text { Aib-Pro-Vxx-Aib-Vxx-Gln-Gln-Pheol }\end{array}$ & [77] \\
\hline BIII & T. longibrachiatum & $\begin{array}{l}\text { Ac-Aib-Ala-Aib-Ala-Aib-Aib-Gln-Aib-Vxx-Aib-Gly-Lxx- } \\
\text { Aib-Pro-Vxx-Aib-Aib-Gln-Gln-Pheol }\end{array}$ & [77] \\
\hline BIV & T. longibrachiatum & $\begin{array}{l}\text { Ac-Aib-Ala-Aib-Ala-Aib-Aib-Gln-Aib-Vxx-Aib-Gly-Lxx- } \\
\text { Aib-Pro-Vxx-Aib-Vxx-Gln-Gln-Pheol }\end{array}$ & [77] \\
\hline $\mathrm{CI}$ & T. atroviride $\mathrm{H} 1 / 226$ & $\begin{array}{l}\text { Ac-Aib-Ala-Aib-Ala-Aib-Ala-Gln-Aib-Vxx-Aib-Gly-Lxx- } \\
\text { Aib-Pro-Vxx-Aib-Aib-Glu-Gln-Pheol }\end{array}$ & [76] \\
\hline $\mathrm{CII}$ & T. atroviride $\mathrm{H} 1 / 226$ & $\begin{array}{l}\text { Ac-Aib-Ala-Aib-Ala-Aib-Ala-Gln-Aib-Vxx-Aib-Gly-Lxx- } \\
\text { Aib-Pro-Vxx-Aib-Vxx-Glu-Gln-Pheol }\end{array}$ & [76] \\
\hline CIII & T. atroviride $\mathrm{H} 1 / 226$ & $\begin{array}{l}\text { Ac-Aib-Ala-Aib-Ala-Aib-Aib-Gln-Aib-Vxx-Aib-Gly-Lxx- } \\
\text { Aib-Pro-Vxx-Aib-Aib-Glu-Gln-Pheol }\end{array}$ & {$[76]$} \\
\hline CIV & T. atroviride $\mathrm{H} 1 / 226$ & $\begin{array}{l}\text { Ac-Aib-Ala-Aib-Ala-Aib-Aib-Gln-Aib-Vxx-Aib-Gly-Lxx- } \\
\text { Aib-Pro-Vxx-Aib-Vxx-Glu-Gln-Pheol }\end{array}$ & {$[76]$} \\
\hline
\end{tabular}

Lxx: Leu/Ile; Vxx: Val/Iva; Lxxol: Leuol/Ileol; Vxxol: Valol/Ivaol.

\subsection{Trichokonins}

Trichoderma koningii and Trichoderma pseudokoningii are common mycoparasitic fungi used to control the fungal phytopathogens of vegetables and fruit trees diseases [78,79]. They can produce trichokonins, which are also found in T. longibrachiatum. Trichokonins have four analogs containing large amounts of Aib and forming a helical conformation with a kink (Pro) (Table 10) $[64,80,81]$. Trichokonins have a broad antibacterial activity against Gram-positive bacteria, especially to Staphylococcus aureus, even multidrug-resistant Staphylococcus aureus. However, trichokonins have no significant effect on Gram-negative bacteria. In addition, trichokonins are highly toxic to hepatocellular carcinoma cells HepG2, lung cancer cells A549 and gastric cancer cells BGC823, leading then to apoptosis [82]. Tichokonin VI is an L-type $\mathrm{Ca}^{2+}$ agonist on cardiac biofilms [83]. In addition, trichokonins also promote antiviral activity of tobacco by inducing the defense response and systemic resistance of tobacco to tobacco mosaic virus (TMV) infection [80].

Table 10. Analogs of trichokonins.

\begin{tabular}{clcc}
\hline Trichokonin & \multicolumn{1}{c}{ Strain } & Composition & Ref. \\
\hline VI & $\begin{array}{c}\text { T. koningii OUDEMANS, } \\
\text { T. pseudokoningii SMF2 } \\
\text { IX }\end{array}$ & $\begin{array}{c}\text { Ac-Aib-Ala-Aib-Ala-Aib-Ala-Gln-Aib-Val-Aib-Gly- } \\
\text { Leu-Aib-Pro-Val-Aib-Aib-Gln-Gln-Pheol } \\
\text { Ac-Aib-Ala-Aib-Ala-Aib-Ala-Gln-Aib-Val-Aib-Gly- } \\
\text { Leu-Aib-Pro-Val-Aib-Iva-Gin-Gln-Pheol }\end{array}$ & [81] \\
Ia & T. koningii OUDEMANS & $\begin{array}{c}\text { Ac-Aib-Ala-Aib-Ala-Aib-Aib-Gln-Aib-Val-Aib-Gly- } \\
\text { Leu-Ala-Pro-Val-Aib-Aib-Gln-Gln-Pheol } \\
\text { Ac-Aib-Gly-Aib-Ala-Aib-Aib-Gln-Aib-Val-Aib-Gly- } \\
\text { Leu-Aib-Pro-Val-Aib-Aib-Gln-Gln-Pheol }\end{array}$ & {$[81,84]$} \\
\hline
\end{tabular}




\subsection{Alamethicins}

Trichoderma viride (NRRL 3199), a BF widely distributed in nature and used to control soil-borne plant diseases [84,85], produces alamethicins with two analogs, B30 and B50 (Table 11) [86]. Each analogs has many derivatives with the absences of the N-terminal six residues or the C-terminal phenylalaninol (pheol) or the substitution of Ala of 6th residue with Aib or Gln of 7th and 19th residues with Glu. Alamethicins rich in Aib and have two Pro near the N-terminal and C-terminal, the N-terminal of the molecule forms a stable $\alpha$-helix and the C-terminal exhibits a variable hydrogen bonding pattern [87]. Alamethicin is often used as a model ion channel for passive diffusion of voltage-gated cation ions $[88,89]$.

Table 11. Analogs of alamethicins

\begin{tabular}{cc}
\hline Alamethicin & Composition \\
\hline F30 & Ac-Aib-Pro-Aib-Ala-Aib-Ala-Gln-Aib-Val-Aib-Gly-Leu-Aib-Pro-Val-Aib-Aib-Glu-Gln-Pheol \\
F50 & Ac-Aib-Pro-Aib-Ala-Aib-Ala-Gln-Aib-Val-Aib-Gly-Leu-Aib-Pro-Val-Aib-Aib-Gln-Gln-Pheol \\
\hline
\end{tabular}

\subsection{Trichotoxins}

Trichotoxins with 18 residues were purified from T. viride and T. asperellum. These compounds are divided into trichotoxin A-40 and A-50 analogs with multiple derivatives (Table 12) [90,91]. The differences among the derivatives are the substitution of Aib/Ala, Gln/Glu and C-terminal Aib/D-Iva and only A-50 contains neutral Gln. Trichotoxins have $\alpha$-helical conformation and form ion channels similar to alamethicin [90]. They cause hemolysis in human erythrocytes at micromolar concentrations and possess cytotoxic activity against $\mathrm{GH}(4) \mathrm{C}(1)$ rat pituitary and Neuro-2a mouse neuroblastoma cells [92,93].

Table 12. Analogs of trichotoxins.

\begin{tabular}{|c|c|c|c|}
\hline Trichotoxin & Strain & Composition & Ref. \\
\hline A-40 & T. viride NRRL 5242 & $\begin{array}{l}\text { Ac-Aib-Gly-Aib-Leu-Aib-Glu-Aib-Aib-Aib-Ala-Aib- } \\
\text { Aib-Pro-Leu-Aib-D-Iva-Gln-Valol }\end{array}$ & {$[90]$} \\
\hline A-40 I & T. viride NRRL 5242 & $\begin{array}{l}\text { Ac-Aib-Gly-Aib-Leu-Aib-Gln-Aib-Aib-Ala-Ala-Aib- } \\
\text { Aib-Pro-Leu-Aib-Aib-Glu-Valol }\end{array}$ & [90] \\
\hline A-40 II & T. viride NRRL 5242 & $\begin{array}{l}\text { Ac-Aib-Gly-Aib-Leu-Aib-Gln-Aib-Aib-Aib-Ala-Ala- } \\
\text { Aib-Pro-Leu-Aib-Aib-Glu-Valol }\end{array}$ & {$[90]$} \\
\hline A-40 III & T. viride NRRL 5242 & $\begin{array}{l}\text { Ac-Aib-Gly-Aib-Leu-Aib-Gln-Aib-Aib-Ala-Ala-Aib- } \\
\text { Aib-Pro-Leu-Aib-D-Iva-Glu-Valol }\end{array}$ & [90] \\
\hline A-40 IV & T. viride NRRL 5242 & $\begin{array}{l}\text { Ac-Aib-Gly-Aib-Leu-Aib-Gln-Aib-Aib-Aib-Ala-Aib- } \\
\text { Aib-Pro-Leu-Aib-Aib-Glu-Valol }\end{array}$ & [90] \\
\hline A- $40 \mathrm{~V}$ & T. viride NRRL 5242 & $\begin{array}{l}\text { Ac-Aib-Gly-Aib-Leu-Aib-Gln-Aib-Aib-Aib-Ala-Aib- } \\
\text { Aib-Pro-Leu-Aib-D-Iva-Glu-Valol }\end{array}$ & {$[90]$} \\
\hline A-40 Va & T. viride NRRL 5242 & $\begin{array}{l}\text { Ac-Aib-Ala-Aib-Leu-Aib-Gln-Aib-Aib-Aib-Ala-Aib- } \\
\text { Aib-Pro-Leu-Aib-Aib-Glu-Valol }\end{array}$ & [90] \\
\hline A-50 E & T. viride NRRL 5242 & $\begin{array}{l}\text { Ac-Aib-Gly-Aib-Leu-Aib-Gln-Aib-Aib-Aib-Ala-Ala- } \\
\text { Aib-Pro-Leu-Aib-Aib-Gln-Valol }\end{array}$ & {$[90]$} \\
\hline $\mathrm{A}-50 \mathrm{~F}$ & T. viride NRRL 5242 & $\begin{array}{l}\text { Ac-Aib-Gly-Aib-Leu-Aib-Gln-Aib-Aib-Ala-Ala-Ala- } \\
\text { Aib-Pro-Leu-Aib-D-Iva-Gln-Valol }\end{array}$ & {$[90]$} \\
\hline A-50 G & T. viride NRRL 5242 & $\begin{array}{l}\text { Ac-Aib-Gly-Aib-Leu-Aib-Gln-Aib-Aib-Aib-Ala-Ala- } \\
\text { Aib-Pro-Leu-Aib-D-Iva-Gln-Valol }\end{array}$ & [90] \\
\hline A-50 H & T. viride NRRL 5242 & $\begin{array}{l}\text { Ac-Aib-Ala-Aib-Leu-Aib-Gln-Aib-Aib-Aib-Ala-Ala- } \\
\text { Aib-Pro-Leu-Aib-D-Iva-Gln-Valol }\end{array}$ & [90] \\
\hline A-50 I & T. viride NRRL 5242 & $\begin{array}{l}\text { Ac-Aib-Gly-Aib-Leu-Aib-Gln-Aib-Aib-Aib-Ala-Aib- } \\
\text { Aib-Pro-Leu-Aib-D-Iva-Gln-Valol }\end{array}$ & {$[90]$} \\
\hline A-50 J & T. viride NRRL 5242 & $\begin{array}{l}\text { Ac-Aib-Ala-Aib-Leu-Aib-Gln-Aib-Aib-Aib-Ala-Aib- } \\
\text { Aib-Pro-Leu-Aib-D-Iva-Gln-Valol }\end{array}$ & {$[90]$} \\
\hline
\end{tabular}

\subsection{Suzukacillins}

Suzukacillins (SZs) are also purified from T. viride [94]. There are two families, suzukacillin A (SZ-A) and suzukacillin B(SZ-B) (Table 13) [94,95]. Suzukacillins exhibit membrane modifying 
and lysing properties similar to those of alamethicin. Suzukacillins inhibit the growth of Bacillus subtilis, Xanthomonas oryzae, Sarcina lutea, Staphylococcus aureus, Mycobacterium phlei, Streptococcus pyogenes, Corynebacterium diphtheriae, Aspergillus niger, Trichophyton fumigatus and Saccharomyces sake at concentrations of 10 to $100 \mu \mathrm{g} / \mathrm{L}$. Suzukacillins also have hemolytic properties against human erythrocytes [93].

Table 13. Analogs of suzukacillins.

\begin{tabular}{|c|c|c|c|}
\hline Suzukacillin & Strain & Composition & Ref. \\
\hline SZ-A1 & T. viride 63C-I & $\begin{array}{l}\text { Ac-Aib-Ala-Aib-Ala-Aib-Ala-Gln-Aib-Vxx-Aib } \\
\text {-Gly-Aib-Aib-Pro-Vxx-Aib-Aib-Gln-Gln-Pheol }\end{array}$ & [94] \\
\hline SZ-A2 & T. viride 63C-I & $\begin{array}{l}\text { Ac-Aib-Ala-Aib-Ala-Aib-Ala-Gln-Aib-Lxx-Aib- } \\
\text { Gly-Aib-Aib-Pro-Vxx-Aib-Aib-Gln-Gln-Pheol }\end{array}$ & [94] \\
\hline SZ-A3 & T. viride 63C-I & $\begin{array}{l}\text { Ac-Aib-Ala-Aib-Ala-Aib-Ala-Gln-Aib-Vxx-Aib- } \\
\text { Gly-Aib-Aib-Pro-Vxx-Aib-Vxx-Gln-Gln-Pheol }\end{array}$ & [94] \\
\hline SZ-A4 & T. viride 63C-I & $\begin{array}{l}\text { Ac-Aib-Ala-Aib-Ala-Aib-Ala-Gln-Aib-Lxx-Aib- } \\
\text { Gly-Aib-Aib-Pro-Vxx-Aib-Vxx-Gln-Gln-Pheol }\end{array}$ & [94] \\
\hline SZ-A5 & T. viride 63C-I & $\begin{array}{l}\text { Ac-Aib-Ala-Aib-Ala-Aib-Aib-Gln-Aib-Lxx-Aib- } \\
\text { Gly-Aib-Aib-Pro-Vxx-Aib-Aib-Gln-Gln-Pheol }\end{array}$ & [94] \\
\hline SZ-A6 & T. viride 63C-I & $\begin{array}{l}\text { Ac-Aib-Ala-Aib-Ala-Aib-Ala-Gln-Aib-Lxx-Aib- } \\
\text { Gly-Aib-Aib-Pro-Vxx-Aib-Aib-Gln-Gln-Pheol }\end{array}$ & [94] \\
\hline SZ-A7 & T. viride 63C-I & $\begin{array}{l}\text { Ac-Aib-Ala-Aib-Ala-Aib-Aib-Gln-Aib-Lxx-Aib- } \\
\text { Gly-Aib-Aib-Pro-Vxx-Aib-Vxx-Gln-Gln-Pheol }\end{array}$ & [94] \\
\hline SZ-A8 & T. viride 63C-I & $\begin{array}{l}\text { Ac-Aib-Ala-Aib-Ala-Aib-Ala-Gln-Aib-Lxx-Aib- } \\
\text { Gly-Aib-Aib-Pro-Vxx-Aib-Vxx-Gln-Gln-Pheol }\end{array}$ & [94] \\
\hline SZ-A9 & T. viride 63C-I & $\begin{array}{l}\text { Ac-Aib-Ala-Aib-Ala-Aib-Ala-Gln-Aib-Lxx-Aib- } \\
\text { Gly-Lxx-Aib-Pro-Vxx-Aib-Aib-Gln-Gln-Pheol }\end{array}$ & [94] \\
\hline SZ-A10a & T. viride 63C-I & $\begin{array}{l}\text { Ac-Aib-Ala-Aib-Ala-Aib-Ala-Gln-Aib-Vxx-Aib- } \\
\text { Gly-Lxx-Aib-Pro-Vxx-Aib-Vxx-Gln-Gln-Pheol }\end{array}$ & [94] \\
\hline SZ-A10b & T. viride 63C-I & $\begin{array}{l}\text { Ac-Aib-Ala-Aib-Ala-Aib-Ala-Gln-Aib-Lxx-Aib- } \\
\text { Gly-Lxx-Aib-Pro-Vxx-Aib-Vxx-Gln-Gln-Pheol }\end{array}$ & [94] \\
\hline SZ-A11a & T. viride 63C-I & $\begin{array}{l}\text { Ac-Aib-Ala-Aib-Ala-Aib-Ala-Gln-Aib-Lxx-Aib- } \\
\text { Gly-Lxx-Aib-Pro-Vxx-Aib-Aib-Gln-Gln-Pheol }\end{array}$ & [94] \\
\hline SZ-A11b & T. viride 63C-I & $\begin{array}{l}\text { Ac-Aib-Ala-Aib-Ala-Aib-Ala-Gln-Aib-Lxx-Aib- } \\
\text { Gly-Lxx-Aib-Pro-Vxx-Aib-Vxx-Gln-Gln-Pheol }\end{array}$ & [94] \\
\hline SZ-A12 & T. viride 63C-I & $\begin{array}{l}\text { Ac-Aib-Ala-Aib-Ala-Aib-Aib-Gln-Aib-Lxx-Aib- } \\
\text { Gly-Lxx-Aib-Pro-Vxx-Aib-Vxx-Gln-Gln-Pheol }\end{array}$ & [94] \\
\hline SZ-A13 & T. viride 63C-I & $\begin{array}{l}\text { Ac-Aib-Ala-Aib-Ala-Aib-Ala-Gln-Aib-Lxx-Aib- } \\
\text { Gly-Lxx-Aib-Pro-Vxx-Aib-Vxx-Gln-Gln-Pheol }\end{array}$ & [94] \\
\hline SZ-B1 & T. viride 63C-I & Ac-Aib-Gln-Vxx-Lxx-Aib-Pro-Lxx-Lxx-Ala-Pro & [95] \\
\hline SZ-B2 & T. viride $63 \mathrm{C}-\mathrm{I}$ & Ac-Aib-Gln-Lxx-Vxx-Ala-Pro-Lxx-Lxx-Aib-Pro-Vxxol & [95] \\
\hline SZ-B3 & T. viride 63C-I & Ac-Aib-Asn-Vxx-Vxx-Aib-Pro-Lxx-Lxx-Aib-Pro-Lxxol & [95] \\
\hline SZ-B4 & T. viride 63C-I & Ac-Ala-Gln-Vxx-Lxx-Aib-Pro-Lxx-Lxx-Aib-Pro-Lxxol & [95] \\
\hline SZ-B5 & T. viride 63C-I & Ac-Ala-Gln-Lxx-Lxx-Aib-Pro-Lxx-Lxx-Aib-Pro-Vxxol & [95] \\
\hline SZ-B6 & T. viride 63C-I & Ac-Ala-Gln-Lxx-Lxx-Aib-Pro-Lxx-Lxx-Aib-Pro-Lxxol- & [95] \\
\hline SZ-B7 & T. viride $63 \mathrm{C}-\mathrm{I}$ & Ac-Ala-Gln-Lxx-Lxx-Aib-Pro-Lxx-Lxx-Aib-Pro-Vxxol & [95] \\
\hline SZ-B8 & T. viride 63C-I & Ac-Aib-Asn-Vxx-Lxx-Aib-Pro-Lxx-Lxx-Aib-Pro-Lxxol & [95] \\
\hline SZ-B9 & T. viride 63C-I & Ac-Aib-Gln-Lxx-Lxx-Aib-Pro-Lxx-Lxx-Aib-Pro-Vxxol & [95] \\
\hline SZ-B10 & T. viride $63 \mathrm{C}-\mathrm{I}$ & Ac-Aib-Gln-Lxx-Vxx-Aib-Pro-Lxx-Lxx-Aib-Pro-Lxxol & [95] \\
\hline SZ-B11 & T. viride 63C-I & Ac-Aib-Asn-Lxx-Lxx-Aib-Pro-Lxx-Lxx-Aib-Pro-Lxxol & [95] \\
\hline SZ-B12 & T. viride 63C-I & Ac-Aib-Gln-Lxx-Lxx-Aib-Pro-Lxx-Lxx-Aib-Pro-Lxxol & [95] \\
\hline SZ-B13 & T. viride 63C-I & Ac-Vxx-Gln-Lxx-Lxx-Aib-Pro-Lxx-Lxx-Aib-Pro-Lxxol & [95] \\
\hline SZ-B14 & T. viride 63C-I & Ac-Aib-Ala-Lxx-Lxx-Aib-Pro-Lxx-Lxx-Aib-Pro-Lxxol & [95] \\
\hline SZ-B15 & T. viride $63 \mathrm{C}-\mathrm{I}$ & Ac-Vxx-Glu-Lxx-Lxx-Aib-Pro-Lxx-Lxx-Aib-Pro-Lxxol & [95] \\
\hline
\end{tabular}

Vxx: Val or Iva; Lxx: Leu or Ile (isovaline); Vxxol: Valol or Ivaol; Lxxol: Leuol or Ileol.

\subsection{Hypomurocins}

Hypomurocins have 13 analogs (A and B series) (Table 14) purified from Hypocrea muroiana which is a BF not only inhibiting various plant diseases, but also promoting plant growth [96], excepting hypomurocin B that is found in Trichoderma harzianum [59,97]. Hypomurocin A has mixed helical conformation containing $\alpha$ - and $3_{10}$-helices, as well as types I and III $\beta$-turn structures to link the helical [98-100]. Hypomurocin B consists of 18 amino acid residues to form the $3_{10}$-helical structure 
rather than by $\alpha$-helical structure [101]. Hypomurocins inhibit Bacillus subtilis and causes hemolysis of rat erythrocytes, moreover, the activity of hypomurocin B is greater than that of hypomurocin A [96].

Table 14. Analogs of hypomurocins.

\begin{tabular}{|c|c|c|c|}
\hline Hypomurocin & Strain & Composition & Ref. \\
\hline A-1 & H. muroiana IFO31288 & Ac-Aib-Gln-Val-Val-Aib-Pro-Leu-Leu-Aib-Pro-Leuol & [96] \\
\hline A-2 & H. muroiana IFO31288 & Ac-D-Iva-Gln-Val-Val-Aib-Pro-Leu-Leu-Aib-Pro-Leuol & [96] \\
\hline A-3 & H. muroiana IFO31288 & Ac-Aib-Gln-Val-Leu-Aib-Pro-Leu-Ile-Aib-Pro-Leuol & [96] \\
\hline A-4 & H. muroiana IFO31288 & Ac-Aib-Gln-Ile-Val-Aib-Pro-Leu-Leu-Aib-Pro-Leuol & [96] \\
\hline A-5 & H. muroiana IFO31288 & Ac-Aib-Gln-Ile-Ile-Aib-Pro-Leu-Leu-Aib-Pro-Leuol & [96] \\
\hline A-5a & H. muroiana IFO31288 & Ac-Aib-Gln-Ile-Leu-Aib-Pro-Leu-Ile-Aib-Pro-Leuol & [96] \\
\hline B-1 & H. muroiana IFO31288 & $\begin{array}{l}\text { Ac-Aib-Ser-Ala-Leu-Aib-Gln-Aib-Val-Aib-Gly-Aib-Aib- } \\
\text { Pro-Leu-Aib-Aib-Gln-Valol }\end{array}$ & [96] \\
\hline B-2 & H. muroiana IFO31288 & $\begin{array}{l}\text { Ac-Aib-Ser-Ala-Leu-Aib-Gln-Aib-Val-Aib-Gly-Aib-Aib- } \\
\text { Pro-Leu-Aib-Aib-Gln-Leuol }\end{array}$ & [96] \\
\hline B-3a & H. muroiana IFO31288 & $\begin{array}{l}\text { Ac-Aib-Ala-Ala-Leu-Aib-Gln-Aib-Val-Aib-Gly-Aib-Aib- } \\
\text { Pro-Leu-Aib-Aib-Gln-Valol }\end{array}$ & [96] \\
\hline B-3b & H. muroiana IFO31288 & $\begin{array}{l}\text { Ac-Aib-Ser-Ala-Leu-Aib-Gln-Iva-Val-Aib-Gly-Aib-Aib- } \\
\text { Pro-Leu-Aib-Aib-Gln-Valol }\end{array}$ & [96] \\
\hline B-4 & H. muroiana IFO31288 & $\begin{array}{l}\text { Ac-Aib-Ser-Ala-Leu-Aib-Gln-Aib-Val-Aib-Gly-Iva-Aib- } \\
\text { Pro-Leu-Aib-Aib-Gln-Valol }\end{array}$ & [96] \\
\hline B-5 & H. muroiana IFO31288 & $\begin{array}{l}\text { Ac-Aib-Ser-Ala-Leu-Aib-Gln-Aib-Val-Aib-Gly-Iva-Aib- } \\
\text { Pro-Leu-Aib-Aib-Gln-Leuol }\end{array}$ & [96] \\
\hline B & T. harzianum & $\begin{array}{l}\text { Ac-Aib-Ser-Ala-Leu-Ala-Gln-Aib-Val-Aib-Gly-Aib-Aib- } \\
\text { Pro-Leu-Aib- Aib-Gln-Valol }\end{array}$ & [59] \\
\hline
\end{tabular}

\subsection{Atroviridins and Neoatroviridins}

Atroviridins are 20-residue peptaibols with three analogs (Table 15) [102,103]. Neoatroviridins are 18-residue peptaibols with four analogs (Table 16) [104]. They are all purified from T. atroviride (F80317), which is a BFs with antifungal activity [104,105]. Atroviridins and neoatroviridins have strong antifungal activity against some plant pathogenic fungi, such as Curvavularia inaqualis, Collectotrichum dematium and Fusarium oxysporum, as well as moderate activity against Verticillium dahliae, Aspergillus niger and Cladosporium sp. They can inhibit Bacillus subtilis and Staphylococcus aureus. However, they show no activity against Gram-negative bacteria and yeast [102]. Moreover, they have significant cytotoxicity against human cancer cell lines, such as prostate (PC-3), melanoma (UACC62) and leukemia (K562) with $\mathrm{IC}_{50}$ values of $2-4 \mu \mathrm{g} / \mathrm{mL}$ [102].

Table 15. Analogs of atroviridins.

\begin{tabular}{cc}
\hline Atroviridin & Composition \\
\hline A & Ac-Aib-Pro-Aib-Ala-Aib-Ala-Gln-Aib-Val-Aib-Gly-Leu-Aib-Pro-Val-Aib-Aib-Gln-Gln-Pheol \\
B & Ac-Aib-Pro-Aib-Ala-Aib-Ala-Gln-Aib-Val-Aib-Gly-Leu-Aib-Pro-Val-Aib-Iva-Gln-Gln-Pheol \\
C & Ac-Aib-Pro-Aib-Ala-Aib-Aib-Gln-Aib-Val-Aib-Gly-Leu-Aib-Pro-Val-Aib-Iva-Gln-Gln-Pheol \\
\hline
\end{tabular}

Table 16. Analogs of neoatroviridins.

\begin{tabular}{cc}
\hline Neoatroviridin & Composition \\
\hline A & Ac-Aib-Gly-Ala-Leu-Aib-Gln-Aib-Leu-Aib-Gly-Iva-Aib-Pro-Leu-Aib-Aib-Gln-Leuol \\
B & Ac-Aib-Gly-Ala-Leu-Iva-Gln-Aib-Leu-Aib-Gly-Iva-Aib-Pro-Leu-Aib-Aib-Gln-Leuol \\
C & Ac-Aib-Gly-Ala-Leu-Aib-Gln-Iva-Leu-Aib-Gly-Iva-Aib-Pro-Leu-Aib-Aib-Gln-Leuol \\
D & Ac-Aib-Gly-Ala-Leu-Iva-Gln-Iva-Leu-Aib-Gly-Iva-Aib-Pro-Leu-Aib-Aib-Gln-Leuol \\
\hline
\end{tabular}

\subsection{Peptaivirin}

Peptaivirins are special peptaibols purified from Trichoderma spp. (KGT142). Peptaivirins have two analogs (Table 17), peptaivirins A and B, which show strong antiviral effects on TMV infection [106]. Peptaivirins are rich in Aib and have an N-terminus of acetylated phenylalanine. 
Table 17. Analogs of peptaivirins.

\begin{tabular}{cc}
\hline Peptaivirin & Composition \\
\hline A & Ac-Phe-Aib-Ala-Aib-Iva-Leu-Gln-Gly-Aib-Aib-Ala-Ala-Aib-Pro-Iva-Aib-Aib-Gln-Trpol \\
B & Ac-Phe-Aib-Ser-Aib-Iva-Leu-Gln-Gly-Aib-Aib-Ala-Ala-Aib-Pro-Iva-Aib-Aib-Gln-Pheol \\
\hline
\end{tabular}

\section{Biosynthesis of L-NRPs}

Non-ribosomal peptides are synthesized by non-ribosomal peptide synthase (NRPS) with multiple modules in some of the largest enzymes found in nature. The modules consist of different domains with specific catalytic activities. The core domains of NRPS include adenylation domain (A domain, recognizing and adenylating the initiation molecule), thiolation domain (T domain, also known as peptidyl carrier protein domain (PCP)) and condensation domain (C domain, catalyzing the corresponding monomers to bind to the new peptide) [8]. In addition to the infrastructure domains (A, T and C domains), NRPSs probably have epimerization domain (E domain), N-methylation domain (M domain) and others to modify the peptide [104]. Finally, there is a thioesterase domain (TE) in bacteria NRPSs or a similar condensation domain (CT domain) in fungal NRPSs to hydrolyze or cyclize the end of the target polypeptide $[107,108]$.

NRPSs are divided into three categories, namely linear, iterative and nonlinear NRPS (Figure 9). The linear NRPSs take C-A-T as the extension module, and the assembly results in the production of linear NRPs or cyclic NRPs. The iterative NRPS has multiple same modules and results in final product of oligopeptides or cyclic NRPs with the multiple residues of the same amino acids. The nonlinear NRPS has other modules $(X)$ and the order of C-A-T is not necessary. It deviates completely from the standard domain organization leading to unexpected products [109]. L-NRPs are mainly synthesized by linear NRPs [109]. The number of modules determines the length of the peptide. Compared with cyclic NRPs, the main difference is whether initiation substrates in A domain have free hydroxyl and amidogen. After hydrolysis in the TE domain, internal esterification or lactam hydrolysis will occur [6]. The biosynthesis process of L-NRPs in biocontrol fungi is complex and few researches have been published, so we take ACV as an example to illustrate the biosynthesis of L-NRPs.

Linear NRPS
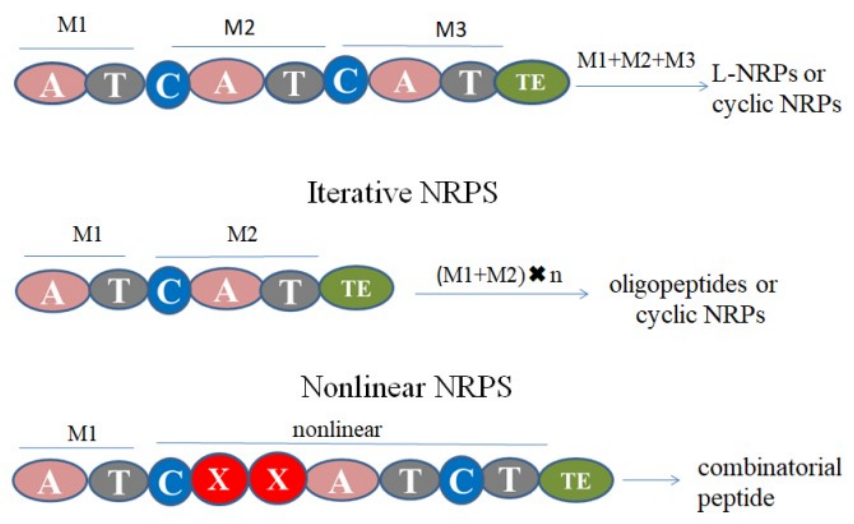

Figure 9. Synthesis of non-ribosomal peptide synthases (NRPSs) types. (M1 module is the initial of synthesis, M2 and M3 are the extension; $X$ in nonlinear NRPS represents other domains).

The PcbAB of ACV synthetase (ACVS) was cloned from P. chrysogenum, it measures $11,500 \mathrm{bp}$ with the open reading frame (ORF) being $11,376 \mathrm{bp}$ and coding for a protein of 3791 aa. The genes, $P c b A B, P c b C$ (encoding cyclase) and $P c b D E$ (encoding penicillin acetyltransferase) form a cluster in the $17 \mathrm{~Kb}$ DNA region to drive penicillin biosynthesis [110]. The ACVS genes of Cephalosporins acremonium and Aspergillus nidulans are similar to those of P. chrysogenum, with more than $60 \%$ similarity [111,112]. ACVS contains ten domains, three modules (M1, M2 and M3), 
in which M3 has the special domains $\mathrm{E}$ and TE domains to conduct epimerization of L-valine and the hydrolysis of ACV (Figure 10) [113]. During biosynthesis, A domain in modules M1 chooses the suitable substrate L- $\alpha$-aminoadipic acid to activate and form an aminoacyl-AMP. Then, it combines with hydrosulfonyl of $\mathrm{T}$ domain to form aminoacyl-S-carrier complex and transferred to modules M2 and form cysteinyl-aminoacyl-S-carrier complex by combining with the activated cysteinyl-S-carrier. Then, it is transferred to M3 and condensated with the activated valinyl-S-carrier into valinyl-cysteinyl-aminoacyl-S-carrier complex. Finally, through intramolecular nucleophilic attacks in TE domain, the L-NRPs $\delta$-(L- $\alpha$-amino hexanedioyl)-L-cysteinyl-D-valine (ACV) is produced (Figure 10).

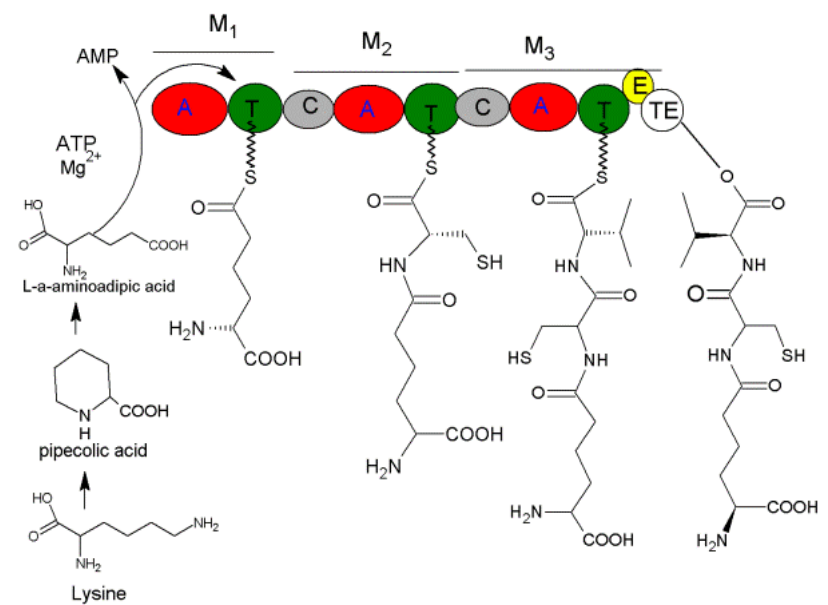

Figure 10. Biosynthesis of ACV.

\section{Discussion}

Although only 22 classes of L-NRPs are found in BFs to date, BFs absolutely have abundant diversity of L-NRPs. First, the BFs L-NRPs have diverse molecular structures, i.e., each class has multiple analogs and numerous derivatives with different configurations and conformations. Second, the BFs L-NRPs have multiple functions because each has diverse bioactivities among of antifungi, antibacteria, antiviruses, insecticides, acaricides, nematicides, herbicides or anticancers. Finally, the BFs L-NRPs have diverse distribution, i.e., one species of BFs has more than one class of L-NRPs, on the other hand, a same L-NRP can exist in different BFs species. For example, Trichoderma harzianum at least has two L-NRPs, harzianins and hypomurocin B, while Trchoderma longibrachiatum produces trichobrachins, trichogins, trilongins and trichokonins. Furthermore, efrapeptins are produced by Tolypocladium niveum, Tolypocladium geodes, Acremonium sp. and Metarhizium anisopliae, while trichokonins can be found in Trichoderma koningii, Trichoderma pseudokoningii and T. longibrachiatum.

Interestingly, more L-NRPs have been found in mycoparasitic fungi than in entomopathogenic fungi, especially in the common entomopathogens, such as Beauveria, Metarhizium and Isaria. The main reason may be related to the L-NRPs characteristics of easy hydrolysis [114]. Insects have many proteases especially in their midguts, so if entomopathogens secrete L-NRPs into insect's body, they will be hydrolyzed soon. However, C-NRPs are difficult to degradation in insects. On the contrary, mycoparasitic fungi usually live in soil and interact with phytopathogens or other microorganisms - in an environment with less proteases. Therefore, the L-NRPs secreted by mycoparaites may persist for a longer time, which has beneficial influences on surrounding microorganisms. The diversity of NRPs is ensured by NRPS through different organizations of domains and modules. The A domains with various structures can select different substrate amino or fatty acids to provide the diverse composition of peptide chain. Undoubtedly, to adapt environments, BFs must take the least costs to obtain the best NRPS genes. Such, the co-evolution of BFs and these target lives leads to less L-NRPs existing in entomopathogenic fungi than in mycoparasitic fungi. 
NRPs as drug resources attract much attention of researchers. BFs L-NRPs have the potential as pesticides and medicines as well. For example, bleomycin has been used to treat cancers [5]. ACV as a precursor compound of penicillin has been concerned for long times [52]. Although NRPs are currently not used in agricultural area, the further studies are valuable. However, the more important is the risks of L-NRPs in BFs. As many L-NRPs are toxic, they can hazard human health and non-target beings once they enter the food chain in the process of agricultural application. Although NRPs produced by BFs have little probability to enter food chain $[2,9,115]$, caution must be exercised. It is necessary that adequate risk assessments are conducted before using BFs.

In conclusion, there are 22 classes L-NRPs found in BFs currently. They have abundant diversity including various structures, functions and distributions. The NRPSs through different compositions of domains and modules accomplish biosynthesis of deferent L-NRPs. Mycoparasitic fungi than entomopathogenic fungi produce more L-NRPs, it is maybe because the co-evolutions of fungi with their hosts lead to NRPSs in these two fungi. BFs L-NRPs have the potential as pesticides and medicines. However, the risks of L-NRPs contaminating foods and environment need be paid more attentions.

Author Contributions: X.N. wrote the study. N.T. revised the MS. Q.H. wrote and revised the MS. All authors have read and agreed to the published version of the manuscript.

Funding: This project was supported by the Key Realm R \& D Program of Guangdong Province (2019B020218009).

Conflicts of Interest: The authors declare no conflict of interest.

\section{References}

1. Zhang, X.F.; Hu, Q.B.; Weng, Q.F. Secondary metabolites (SMs) of Isaria cicadae and Isaria tenuipes. RSC Adv. 2019, 9, 172-184. [CrossRef]

2. Weng, Q.F.; Zhang, X.F.; Chen, W.; Hu, Q.B. Secondary metabolites and the risks of Isaria fumosorosea and Isaria farinosa. Molecules 2019, 24, 664. [CrossRef]

3. Marian, M.; Shimizu, M. Improving performance of microbial biocontrol agents against plant diseases. J. Gen. Plant Pathol. 2019, 85, 329-336. [CrossRef]

4. Silva, R.N.; Monteiro, V.N.; Steindorff, A.S.; Gomes, E.V.; Noronha, E.F.; Ulhoa, C.J. Trichoderma/pathogen/plant interaction in pre-harvest food security. Fungal Biol. 2019, 123, 565-583. [CrossRef] [PubMed]

5. Süssmuth, R.D.; Mainz, A. Nonribosomal peptide synthesis-principles and prospects. Angew. Chem. Int. Ed. Engl. 2017, 56, 3770-3821. [CrossRef]

6. Xue, G.; Stuart, W.H.; Brian, D.A.; Peng, W.; Linda, P.V.; Christopher, T.W.; Yi, T. Cyclization of fungal nonribosomal peptides by a terminal condensation-like domain. Nat. Chem. Biol. 2012, 8, 823-830.

7. Matthias, S.; Alan, T.; Mohamed, A.M. Nonribosomal peptide synthetases: Structures and dynamics. Curr. Opin. Struct. Biol. 2010, 20, 234-240.

8. Bradley, R.M.; Andrew, M.G. Structural biology of nonribosomal peptide synthetases. Methods Mol. Biol. 2016, 1401, 3-29.

9. Hu, Q.; Dong, T. Chapter 8 Non-ribosomal Peptides from Entomogenous Fungi. In Biocontrol of Lepidoperan Pests-Use of Soil Microbes and their Metabolites; Sree, K.S., Varma, A., Eds.; Springer: Cham, Switzerland, 2015; Volume 43, pp. 169-206. [CrossRef]

10. Krasnoff, S.B.; Reategui, R.F.; Wagenaar, M.M.; Gloer, J.B.; Gibson, D.M. Cicadapeptins I and II: New aib-containing peptides from the entomopathogenic fungus Cordyceps heteropoda. J. Nat. Prod. 2005, 68, 50-55. [CrossRef]

11. Molnár, I.; Gibson, D.M.; Krasnoff, S.B. Secondary metabolites from entomopathogenic Hypocrealean fungi. Nat. Prod. Rep. 2010, 27, 1241-1275. [CrossRef]

12. Thongtan, J.; Saenboonrueng, J.; Rachtawee, P.; Isaka, M. An antimalarial tetrapeptide from the entomopathogenic fungus Hirsutella sp. BCC 1528. J. Nat. Prod. 2006, 69, 713-714. [CrossRef] [PubMed]

13. Martinez, A.F.C.; Moraes, L.A.B. Liquid chromatography-tandem mass spectrometry characterization of five new leucinostatins produced by Paecilomyces lilacinus CG-189. J. Antibiot. 2015, 68, 178-184. [CrossRef] [PubMed] 
14. Radics, L.; Kajtarperedy, M.; Casinovi, C.G.; Rossi, C.; Ricci, M.; Tuttobello, L. Leucinostatins H and K, two novel peptide antibiotics with tertiary amine-oxide terminal group from Paecilomyces marquandii isolation, structure and biological activity. J. Antibiot. 1987, 40, 714-716. [PubMed]

15. Strobel, G.A.; Torczynski, R.; Bollon, A. Acremonium sp.: A leucinostatin A producing endophyte of European yew (Taxus baccata). Plant Sci. 1997, 128, 97-108.

16. Shima, A.; Fukushima, K.; Arai, T.; Terada, H. Dual inhibitory effects of the peptide antibiotics leucinostatins on oxidative phosphorylation in mitochondria. Cell Struct. Funct. 1990, 15, 53-58. [CrossRef] [PubMed]

17. Park, J.O.; Hargreaves, J.R.; Mcconville, E.J.; Stirling, G.R.; Ghisalberti, E.L.; Sivasithamparam, K. Production of leucinostatins and nematicidal activity of Australian isolates of Paecilomyces lilacinus (Thom) Samson. Lett. Appl. Microbiol. 2010, 38, 271-276. [CrossRef]

18. Manabu, K.; Hiroyuki, I.; Shun-Ichi, O.; Tohru, M.; Isao, M.; Daishiro, I. Leucinostatin A inhibits prostate cancer growth through reduction of insulin-like growth factor-I expression in prostate stromal cells. Int. J. Cancer 2010, 126, 810-818.

19. Krasnoff, S.B.; Gupta, S. Identification and directed biosynthesis of efrapeptins in the fungus Tolypocladium geodes gams (Deuteromycotina: Hyphomycetes). J. Chem. Ecol. 1991, 17, 1953-1962. [CrossRef]

20. Krasnoff, S.B.; Gupta, S.; Leger, R.J.S.; Renwick, J.A.A.; Roberts, D.W. Antifungal and insecticidal properties of the efrapeptins: Metabolites of the fungus Tolypocladium niveum. J. Invertebr. Pathol. 1991, 58, 180-188. [CrossRef]

21. Meyling, N.V.; Schmidt, N.M.; Eilenberg, J. Occurrence and diversity of fungal entomopathogens in soils of low and high Arctic Greenland. Polar Biol. 2012, 35, 1439-1445. [CrossRef]

22. Boot, C.M.; Amagata, T.; Tenney, K.; Compton, J.E.; Pietraszkiewicz, H.; Valeriote, F.A.; Crews, P. Four classes of structurally unusual peptides from two marine-derived fungi: Structures and bioactivities. Tetrahedron 2007, 63, 9903-9914. [CrossRef] [PubMed]

23. Papathanassiu, A.E.; MacDonald, N.J.; Emlet, D.R.; Hong, A.V. Antitumor activity of efrapeptins, alone or in combination with 2-deoxyglucose, in breast cancer in vitro and in vivo. Cell Stress Chaperon. 2011, 16, 181-193. [CrossRef] [PubMed]

24. Abrahams, J.P.; Buchanan, S.K.; Raaij, M.J.V.; Fearnley, I.M.; Walker, J.E. The structure of bovine F1-ATPase complexed with the peptide antibiotic efrapeptin. Proc. Natl. Acad. Sci. USA 1996, 93, 9420-9424. [CrossRef] [PubMed]

25. Papathanassiu, A.E.; MacDonald, N.J.; Bencsura, A.; Vu, H.A. F1F0-ATP synthase functions as a co-chaperone of HSP90-Substrate protein complexes. Biochem. Biophys. Res. Commun. 2006, 345, 420-429. [CrossRef]

26. Laurence, H.P. Review: The HSP90 molecular chaperone-An enigmatic ATPase. Biopolymers 2016, 105, 594-607.

27. Bandani, A.R.; Amiri, B.; Butt, T.M.; Gordon-Weeks, R. Effects of efrapeptin and destruxin, metabolites of entomogenous fungi, on the hydrolytic activity of a vacuolar type ATPase identified on the brush border membrane vesicles of Galleria mellonella midgut and on plant membrane bound hydrolytic enzymes. $B B A$ Biomembranes 2001, 1510, 367-377. [CrossRef]

28. Yoichi, H.; Yuki, H.; Takashi, K.; Kaneo, K.; Kyoko, A.; Yoshikazu, S.; Kazuo, S.Y. Efrapeptin J, a new down-regulator of the molecular chaperone GRP78 from a marine Tolypocladium sp. J. Antibiot. 2008, 61, 365-371.

29. Szekeres, A.; Leiteb, B.; Kredics, L.; Antal, Z.; Hatvani, L.; Manczinger, L.; Vágvölgyi, C. Peptaibols and related peptaibiotics of Trichoderma. Acta Microbiol. Immunol. Hung. 2005, 52, 137-168. [CrossRef]

30. Wang, Z.; Wang, G.S. APD: The antimicrobial peptide database. Nucleic Acids Res. 2004, 32, 590-592. [CrossRef]

31. Zhao, P.; Xue, Y.; Li, X.; Li, J.; Zhao, Z.; Quan, C.; Gao, W.; Zu, X.; Bai, X.; Feng, S. Fungi-derived lipopeptide antibiotics developed since 2000. Peptides 2019, 113, 52-65. [CrossRef]

32. Higashimoto, Y.; Kodama, H.; Jelokhani-Niaraki, M.; Kato, F.; Kondo, M. Structure-function relationship of model Aib-containing peptides as ion transfer intermembrane templates. J. Biochem. 1999, 125, 705-712. [CrossRef]

33. Miick, S.M.; Martinez, G.V.; Fiori, W.R.; Todd, A.P.; Millhauser, G.L. Short alanine-based peptides may form $3_{10}$-helices and not $\alpha$-helices in aqueous solution. Nature 1992, 359, 653-655. [CrossRef] [PubMed]

34. Chugh, J.K.; Wallace, B.A. Peptaibols: Models for ion channels. Biochem. Soc. Trans. 2001, 29, 565-570. [CrossRef] [PubMed] 
35. Poirier, L.; Quiniou, F.; Ruiz, N.; Montagu, M.; Amiard, J.C.; Pouchus, Y.F. Toxicity assessment of peptaibols and contaminated sediments on Crassostrea gigas embryos. Aquat. Toxicol. 2007, 83, 254-262. [CrossRef] [PubMed]

36. Peltola, J.; Ritieni, A.; Mikkola, R.; Grigoriev, P.A.; Pócsfalvi, G.; Andersson, M.A.; Salkinojasalonen, M.S. Biological Effects of Trichoderma harzianum Peptaibols on mammalian Cells. Appl. Environ. Microbiol. 2004, 70, 4996-5004. [CrossRef] [PubMed]

37. Unkles, S.E.; Marriott, C.; Kinghorn, J.R.; Panter, C.; Blackwell, A. Efficacy of the entomopathogenic fungus, Culicinomyces clavisporus against larvae of the biting midge, Culicoides nubeculosus (Diptera: Ceratopogonidae). Biocontrol Sci. Technol. 2004, 14, 397-401. [CrossRef]

38. Haiyin, H.; Janso, J.E.; Hui, Y.; Yang; Bernan, V.S.; Lin, S.L.; Ker, Y. Culicinin D, an antitumor peptaibol produced by the fungus Culicinomyces clavisporus, strain LL-12I252. J. Nat. Prod. 2006, 69, 736-741.

39. Kebede, B.; Wrigley, S.K.; Prashar, A.; Rahlff, J.; Wolf, M.; Reinshagen, J.; Gribbon, P.; Imhoff, J.F.; Silber, J.; Labes, A. Establishing the secondary metabolite profile of the marine fungus:Tolypocladium geodes sp. MF458 and subsequent optimisation of bioactive secondary metabolite production. Mar. Drugs 2017, 15, 84. [CrossRef]

40. Tsantrizos, Y.S.; Pischos, S.; Sauriol, F. Structural assignment of the peptide antibiotic LP237-F8, a metabolite of $\backslash \mathbf{r}$, Tolypocladium geodes. J. Org. Chem. 1996, 61, 2118-2121. [CrossRef]

41. Tsantrizos, Y.S.; Pischos, S.; Sauriol, F.; Widden, P. Peptaibol metabolites of Tolypocladium geodes. Can. J. Chem. 1996, 74, 165-172. [CrossRef]

42. Mario, R.; Alessandro, M.; Cristina, P.; Fernando, F.; Stefano, M.; Evaristo, P.; José Antonio, G.; Maria Dolores, D.D.V.; Carlos, C.; Claudio, T. Lipopeptaibol metabolites of tolypocladium geodes: Total synthesis, preferred conformation, and membrane activity. Chem. Eur. J. 2003, 9, 3567-3576.

43. Kimonyo, A.; Brückner, H. Sequences of metanicins, 20-residue peptaibols from the ascomycetous fungus CBS 597.80. Chem. Biodivers. 2013, 10, 813-826. [CrossRef] [PubMed]

44. Tahlan, K.; Moore, M.A.; Jensen, S.E. $\delta$-(L- $\alpha$-aminoadipyl)-L-cysteinyl-D-valine synthetase (ACVS): Discovery and perspectives. J. Ind. Microbiol. Biotechnol. 2016, 44, 517-524. [CrossRef] [PubMed]

45. Barbara, T.; Andres, B.; Thomas, B.; Urs, G.; Sonia, J.; Christina, R.; Lucius, T. An aqueous extract of the dry mycelium of Penicillium chrysogenum induces resistance in several crops under controlled and field conditions. Eur. J. Plant Pathol. 2006, 114, 185-197.

46. Konstantopoulou, M.A.; Mazomenos, B.E. Evaluation of Beauveria bassiana and B. brongniartii strains and four wild-type fungal species against adults of Bactrocera oleae and Ceratitis capitata. Biocontrol 2005, 50, $293-305$. [CrossRef]

47. Etchegaray, A.; Dieckmann, R.; Kennedy, J.; Turner, G.; Von, D.H. ACV synthetase: Expression of amino acid activating domains of the Penicillium chrysogenum enzyme in Aspergillus nidulans. Biochem. Biophys. Res. Commun. 1997, 237, 166-169. [CrossRef]

48. Hoskins, J.A.; O'Callaghan, N.; Queener, S.W.; Cantwell, C.A.; Wood, J.S.; Chen, V.J.; Skatrud, P.L. Gene disruption of the $p c b A B$ gene encoding ACV synthetase in Cephalosporium acremonium. Curr. Genet. 1990, 18, 523-530. [CrossRef]

49. Jensen, S.E.; Westlake, D.W.S.; Bowers, R.J.; Ingold, C.F.; Jouany, M.; Lyubechansky, L.; Wolfe, S. Penicillin formation by cell-free extracts of Streptomyces clavuligerus. Behaviour of aminoadipyl-modified analogs of the natural peptide precursor $\delta$-(L- $\alpha$-aminoadipyl)-L-cysteinyl-D-valine (ACV). Can. J. Chem. 2011, 62, 2712-2720. [CrossRef]

50. Sundaravadivelan, C.; Padmanabhan, M.N. Effect of mycosynthesized silver nanoparticles from filtrate of Trichoderma harzianum against larvae and pupa of dengue vector Aedes aegypti L. Environ. Sci. Pollut. Res. Int. 2014, 21, 4624-4633. [CrossRef]

51. Lucaciu, M.; Rebuffat, S.; Goulard, C.; Duclohier, H.; Molle, G.; Bodo, B. Interaction of the 14-residue peptaibols, harzianins $\mathrm{HC}$, with lipid bilayers: Permeability modifications and conductance properties. $B B A$ Biomembranes 1997, 1323, 85-96. [CrossRef]

52. Ségalas, I.; Prigent, Y.; Davoust, D.; Bodo, B.; Rebuffat, S. Characterization of a type of $\beta$-bend ribbon spiral generated by the repeating (Xaa-Yaa-Aib-Pro) motif: The solution structure of harzianin HC IX, a 14-residue peptaibol forming voltage-dependent ion channels. Biopolymers 1999, 50, 71-85. [CrossRef]

53. Leclerc, G.; Goulard, C.; Bodo, B.; Rebuffat, S. Directed biosynthesis of peptaibol antibiotics in two Trichoderma strains I. fermentation and isolation. J. Antibiot. 1998, 51, 178-183. [CrossRef] 
54. Augeven-Bour, I.; Rebuffat, S.; Auvin, C.; Goulard, C.; Bodo, B. Harzianin HB I, an 11-Residue peptaibol from Trichoderma harzianum: Isolation, sequence, solution synthesis and membrane activity. J. Chem. Soc. Perkin Trans. 1997, 1, 1587-1594. [CrossRef]

55. Putzu, M.; Kara, S.; Afonin, S.; Grage, S.L.; Bordessa, A.; Chaume, G.; Brigaud, T.; Ulrich, A.S.; Kubaå, T. Structural behavior of the peptaibol harzianin HK VI in a DMPC bilayer: Insights from MD simulations. Biophys. J. 2017, 112, 2602-2614. [CrossRef] [PubMed]

56. Rebuffat, S.; Duclohier, H.; Auvin-Guette, C.; Molle, G.; Spach, G.; Bodo, B. Membrane-modifying properties of the pore-forming peptaibols saturnisporin SA IV and harzianin HA V. FEMS Microbiol. Lett. 1992, 105, 51-60. [CrossRef] [PubMed]

57. Duval, D. Isolation and sequence analysis of the peptide antibiotics trichorzins PA from Trichoderma harzianum. J. Chem. Soc. Perkin Trans. 1997, 1, 2147-2154. [CrossRef]

58. Béven, L.; Duval, D.; Rebuffat, S.; Riddell, F.G.; Bodo, B.; Wróblewski, H. Membrane permeabilisation and antimycoplasmic activity of the 18-residue peptaibols, trichorzins PA. BBA Biomembranes 1998, 1372, 78-90. [CrossRef]

59. Aric, W.; Darlene, G.; Bi-Wen, X.; Christophe, G.; Sylvie, R.; Ebbole, D.J.; Bernard, B.; Charles, K. Identification of peptaibols from Trichoderma virens and cloning of a peptaibol synthetase. J. Biol. Chem. 2002, 277, 20862-20868.

60. Duval, D.; Cosette, P.; Rebuffat, S.; Duclohier, H.; Bodo, B.; Molle, G. Alamethicin-like behaviour of new 18-residue peptaibols, trichorzins PA. role of the C-terminal amino-alcohol in the ion channel forming activity. BBA Biomembranes 1998, 1369, 309-319. [CrossRef]

61. Sreenivasaprasad, S.; Manibhushanrao, K. Antagonistic potential of Gliocladium virens and Trichoderma longibrachiatum to phytopathogenic fungi. Mycopathologia 1990, 109, 19-26. [CrossRef]

62. Ghosh, S.K.; Pal, S. Entomopathogenic potential of Trichoderma longibrachiatum and its comparative evaluation with malathion against the insect pest Leucinodes orbonalis. Environ. Monit. Assess. 2016, 188, 37. [CrossRef] [PubMed]

63. Zhang, S.W.; Gan, Y.T.; Xu, B.L. Efficacy of Trichoderma longibrachiatum in the control of Heterodera avenae. Biocontrol 2014, 59, 319-331. [CrossRef]

64. Mohamed-Benkada, M.; Pouchus, Y.F.; Vérité, P.; Pagniez, F.; Caroff, N.; Ruiz, N. Identification and biological activities of long-chain peptaibols produced by a marine-derived strain of Trichoderma longibrachiatum. Chem. Biodivers. 2016, 13, 521-530. [CrossRef] [PubMed]

65. Leclerc, G. Sequences and antimycoplasmic properties of longibrachins LGB II and LGB III, two novel 20-residue peptaibols from Trichoderma longibrachiatum. J. Nat. Prod. 2001, 64, 164-170. [CrossRef]

66. Cosette, P.; Rebuffat, S.; Bodo, B.; Molle, G. The ion-channel activity of longibrachins LGA I and LGB II: Effects of pro-2/Ala and gln-18/Glu substitutions on the alamethicin voltage-gated membrane channels. $B B A$ Biomembranes 1999, 1461, 113-122. [CrossRef]

67. Nicolas, R.; Karina, P.; Marieke, V.; Isabelle, K.; Joseph, B.; Zouher, A.; Jean-François, B.; Olivier, G.; Yves François, P. Enhancement of domoic acid neurotoxicity on Diptera larvae bioassay by marine fungal metabolites. Toxicon 2010, 55, 805-810.

68. Krause, C.; Kirschbaum, J.; Brückner, H. Peptaibiomics: Microheterogeneity, dynamics, and sequences of trichobrachins, peptaibiotics from Trichoderma parceramosum Bissett (T. longibrachiatum Rifai). Chem. Biodivers. 2010, 38, 1083-1102. [CrossRef]

69. Mustapha, M.B.; Monique, M.; Jean-François, B.; Florence, M.; Philippe, V.; Michèle, D.; John, B.; Yves François, P. New short peptaibols from a marine Trichoderma strain. Rapid Commun. Mass Spectrom. 2006, 20, 1176-1180.

70. Auvin-Guette, C.; Rebuffat, S.; Prigent, Y.; Bodo, B. Trichogin A IV, an 11-residue lipopeptaibol from Trichoderma longibrachiatum. J. Am. Chem. Soc. 1992, 114, 2170-2174. [CrossRef]

71. Salnikov, E.S.; Zotti, M.D.; Bobone, S.; Mazzuca, C.; Bechinger, B. Trichogin GA IV alignment and oligomerization in phospholipid bilayers. ChemBioChem 2019, 20, 2141-2150. [CrossRef]

72. Toniolo, C.; Crisma, M.; Formaggio, F.; Peggion, C.; Epand, R.F.; Epand, R.M. Lipopeptaibols, a novel family of membrane active, antimicrobial peptides. Cell. Mol. Life Sci. 2001, 58, 1179-1188. [CrossRef] [PubMed]

73. De, Z.M.; Biondi, B.; Peggion, C.; Formaggio, F.; Park, Y.; Hahm, K.S.; Toniolo, C. Trichogin GA IV: A versatile template for the synthesis of novel peptaibiotics. Org. Biomol. Chem. 2012, 10, 1285-1299. 
74. Marta, D.Z.; Barbara, B.; Fernando, F.; Claudio, T.; Lorenzo, S.; Yoonkyung, P.; Kyung-Soo, H. Trichogin GA IV: An antibacterial and protease-resistant peptide. J. Pept. Sci. 2010, 15, 615-619.

75. Regina, T.; Giulia, M.; Marta, D.Z.; Cristina, P.; Barbara, B.; Fernando, F.; Emanuele, P. The peculiar N- and C-termini of trichogin GA IV are needed for membrane interaction and human cell death induction at doses lacking antibiotic activity. BBA Biomembranes 2015, 1848, 134-144.

76. Röhrich, C.R.; Iversen, A.; Jaklitsch, W.M.; Voglmayr, H.; Vilcinskas, A.; Nielsen, K.F.; Thrane, U.; Von, D.H.; Brückner, H.; Degenkolb, T. Screening the biosphere: The fungicolous fungus Trichoderma phellinicola, a prolific source of hypophellins, new 17-, 18-, 19-, and 20-residue peptaibiotics. Chem. Biodivers. 2013, 10, 787-812. [CrossRef] [PubMed]

77. Mikkola, R.; Andersson, M.A.; Kredics, L.; Grigoriev, P.A.; Sundell, N.; Salkinoja-Salonen, M.S. 20-Residue and 11-residue peptaibols from the fungus Trichoderma longibrachiatum are synergistic in forming $\mathrm{Na}+\mathrm{K}+$ -permeable channels and adverse action towards mammalian cells. FEBS J. 2012, 279, 4172-4190. [CrossRef] [PubMed]

78. Metcalf, D.A.; Wilson, C.R. The process of antagonism of Sclerotium cepivorum in white rot affected onion roots by Trichoderma koningii. Plant Pathol. 2001, 50, 249-257. [CrossRef]

79. Mukherjee, P.K.; Sherkhane, P.D.; Murthy, N.B. Induction of stable benomyl-tolerant phenotypic mutants of Trichoderma pseudokoningii MTCC 3011, and their evaluation for antagonistic and biocontrol potential. Indian J. Exp. Biol. 1999, 37, 710-712.

80. Luo, Y.; Zhang, D.D.; Dong, X.W.; Zhao, P.B.; Chen, L.L.; Song, X.Y.; Wang, X.J.; Chen, X.L.; Shi, M.; Zhang, Y.Z. Antimicrobial peptaibols induce defense responses and systemic resistance in tobacco against tobacco mosaic virus. FEMS Microbiol. Lett. 2010, 313, 120-126. [CrossRef]

81. Huang, Q.; Tezuka, Y.; Hatanaka, Y.; Kikuchi, T.; Nishi, A.; Tubaki, K. Studies on metabolites of mycoparasitic fungi. IV. Minor peptaibols of Trichoderma koningii. Chem. Pharm. Bull. 1995, 43, 1663-1667. [CrossRef]

82. Song, X.Y.; Shen, Q.T.; Xie, S.T.; Chen, X.L.; Sun, C.Y.; Zhang, Y.Z. Broad-spectrum antimicrobial activity and high stability of trichokonins from Trichoderma koningii SMF2 against plant pathogens. FEMS Microbiol. Lett. 2006, 260, 119-125.

83. Huang, Q.; Tezuka, Y.; Kikuchi, T.; Momose, Y. Trichokonin VI, a new $\mathrm{Ca}^{2+}$ channel agonist in bullfrog cardiac myocytes. Eur. J. Pharmacol. 1994, 271, 5-6. [CrossRef]

84. Ubalua, A.O.; Oti, E. Antagonistic properties of Trichoderma viride on post harvest cassava root rot pathogens. Afr. J. Biotechnol. 2007, 6, 2447-2450. [CrossRef]

85. Naglot, A.; Goswami, S.; Rahman, I.; Shrimali, D.D.; Yadav, K.K.; Gupta, V.K.; Rabha, A.J.; Gogoi, H.K.; Veer, V. Antagonistic potential of native Trichoderma viride Strain against potent tea fungal pathogens in north east India. Plant Pathol. J. 2015, 31, 278-289. [CrossRef] [PubMed]

86. Psurek, A.; Neususs, C.; Pelzing, M.; Scriba, G.K.E. Analysis of the lipophilic peptaibol alamethicin by nonaqueous capillary electrophoresis-electrospray ionization-mass spectrometry. Electrophoresis 2005, 26, 4368-4378. [CrossRef]

87. Cafiso, D.S. Alamethicin: A peptide model for voltage gating and protein-membrane interactions. Ann. Rev. Biophys. 1994, 23, 141-165.

88. Tieleman, D.P.; Berendsen, H.J.C.; Mark, S.P.S. An alamethicin channel in a lipid bilayer: Molecular dynamics simulations. Biophys. J. 1999, 76, 1757-1769. [CrossRef]

89. Matsue, T.; Shiku, H.; Yamada, H.; Uchida, I. Permselectivity of voltage-gated alamethicin ion channel studied by microamperometry. J. Phys. Chem. C 2002, 98, 394-402. [CrossRef]

90. Chugh, J.K.; Brückner, H.; Wallace, B.A. Model for a helical bundle channel based on the high-resolution crystal structure of trichotoxinA 50E. Biochemistry 2002, 41, 12934-12941. [CrossRef]

91. Chutrakul, C.; Alcocer, M.; Bailey, K.; Peberdy, J.F. The production and characterisation of trichotoxin peptaibols, by Trichoderma asperellum. Chem. Biodivers. 2008, 5, 1694-1706. [CrossRef]

92. Schock, T.B.; Huncik, K.; Beauchesne, K.R.; Villareal, T.A.; Moeller, P.D. Identification of trichotoxin, a novel chlorinated compound associated with the bloom forming Cyanobacterium, Trichodesmium thiebautii. Environ. Sci. Technol. 2011, 45, 7503-7509. [CrossRef] [PubMed]

93. Duclohier, H.; Alder, G.M.; Bashford, C.L.; Brückner, H.; Chugh, J.K.; Wallace, B.A. Conductance Studies on Trichotoxin_A50E and Implications for Channel Structure. Biophys. J. 2004, 87, 1705-1710. [CrossRef]

94. Krause, C.; Kirschbaum, J.; Jung, G.; Brückner, H. Sequence diversity of the peptaibol antibiotic suzukacillin-A from the mold Trichoderma viride. J. Pept. Sci. 2005, 12, 321-327. [CrossRef] [PubMed] 
95. Röhrich, C.R.; Vilcinskas, A.; Brückner, H.; Degenkolb, T. The sequences of the eleven-residue peptaibiotics: Suzukacillins-B. Chem. Biodivers. 2013, 10, 827-837. [CrossRef] [PubMed]

96. Becker, D.; Kiess, M.; Brückner, H. Structures of peptaibol antibiotics hypomurocin A and B from the Ascomycetous fungus Hypocrea muroiana hino et katsumoto. Eur. J. Org. Chem. 1997, 1997, 767-772. [CrossRef]

97. Röhrich, C.R.; Jaklitsch, W.M.; Voglmayr, H.; Iversen, A.; Vilcinskas, A.; Nielsen, K.F.; Thrane, U.; von Döhren, H.; Brückner, H.; Degenkolb, T. Front line defenders of the ecological niche: Screening the structural diversity of peptaibiotics from saprotrophic and fungicolous Trichoderma/Hypocreaspecies. Fungal Divers. 2014, 69, 117-146. [CrossRef]

98. Pradeille, N.; Zerbe, O.; Möhle, K.; Linden, A.; Heimgartner, H. The first total synthesis of the peptaibol hypomurocin A1 and its conformation analysis: An application of the 'Azirine/Oxazolone Method'. Chem. Biodivers. 2005, 2, 1127-1152. [CrossRef]

99. Pradeille, N.; Tzouros, M.; Möhle, K.; Linden, A.; Heimgartner, H. Total synthesis of the peptaibols hypomurocin A3 and hypomurocin A5, and their conformation analysis. Chem. Biodivers. 2012, 9, 2528-2558. [CrossRef]

100. Zoltán, N.; János, H.; Balázs, L. In silico conformational analysis of the short-sequence hypomurocin A peptides. Int. J. Pept. 2015, 2015, 281065. [CrossRef]

101. Horváth, J.; Násztor, Z.; Bartha, F.; Bogár, F.; Leitgeb, B. Characterizing the structural and folding properties of long-sequence hypomurocin B peptides and their analogs. Biopolymers 2016, 106, 645-657. [CrossRef]

102. Seung-Uk, O.H.; Yun, B.S.; Sang-Jun, L.; Jung-Han, K.; Ick-Han, Y. Atroviridins A C and neoatroviridins A D, novel peptaibol antibiotics produced by Trichoderma atroviride F80317 I. taxonomy, fermentation, isolation and biological activities. J. Antibiot. 2002, 55, 211.

103. Oh, S.U.; Lee, S.J.; Kim, J.H.; Yoo, I.-D. Structural elucidation of new antibiotic peptides, atroviridins A, B and C from Trichoderma atroviride. Tetrahedron Lett. 2010, 41, 61-64. [CrossRef]

104. Carver, C.E.; Pitt, D.; Rhodes, D.J. Aetiology and biological control of Fusarium wilt of pinks (Dianthus caryophyllus) using Trichoderma aureoviride. Plant Pathol. 1996, 45, 618-630. [CrossRef]

105. Kandasamy, S.K.; Myeong-Hyeon, W. Isolation and molecular identification of Trichoderma species from wetland soil and their antagonistic activity against phytopathogens. Physiol. Mol. Plant Pathol. 2020, 109, 101458. [CrossRef]

106. Yun, B.S.; Yoo, I.D.; Kim, Y.H.; Kim, Y.S.; Lee, S.J.; Kim, K.S.; Yeo, W.H. Peptaivirins A and B, two new antiviral peptaibols against TMV infection. Tetrahedron Lett. 2000, 41, 1429-1431. [CrossRef]

107. Walsh, C.T.; Chen, H.; Keating, T.A.; Hubbard, B.K.; Losey, H.C.; Luo, L.; Marshall, C.G.; Miller, D.A.; Patel, H.M. Tailoring enzymes that modify nonribosomal peptides during and after chain elongation on NRPS assembly lines. Curr. Opin. Chem. Biol. 2001, 5, 525-534. [CrossRef]

108. Zhang, J.; Liu, N.; Cacho, R.A.; Gong, Z.; Liu, Z.; Qin, W.; Tang, C.; Tang, Y.; Zhou, J. Structural basis of nonribosomal peptide macrocyclization in fungi. Nat. Chem. Biol. 2016, 12, 1001-1003. [CrossRef]

109. Mootz, H.D.; Schwarzer, D.; Marahiel, M.A. Ways of assembling complex natural products on modular nonribosomal peptide synthetases. ChemBioChem 2002, 3, 490-504. [CrossRef]

110. Axel, A.B.; Petra, S.; Qusai, A.A.; Alexander, G.; Hans, P.; André, T. Regulation of penicillin biosynthesis in filamentous fungi. Adv. Biochem. Eng. Biot. 2004, 88, 45-90.

111. Gutierrez, S.; Diez, B.; Montenegro, E.; Martin, J.F. Characterization of the Cephalosporium acremonium pcbAB gene encoding alpha-aminoadipyl-cysteinyl-valine synthetase, a large multidomain peptide synthetase: Linkage to the $p c b C$ gene as a cluster of early cephalosporin biosynthetic genes and evidence of multiple functional domains. J. Bacteriol. 1991, 173, 2354-2365.

112. MacCabe, A.P.; Liempt, H.V.; Palissa, H.; Unkles, S.E.; Riach, M.B.; Pfeifer, E.; Dohren, H.V.; Kinghorn, J.R. Delta-(L-alpha-aminoadipyl)-L-cysteinyl-D-valine synthetase from Aspergillus nidulans. molecular characterization of the acvA gene encoding the first enzyme of the penicillin biosynthetic pathway. J. Biol. Chem. 1991, 266, 12646-12654. [PubMed]

113. Wu, X.; García-Estrada, C.; Vaca, I.; Martín, J.F. Motifs in the C-terminal region of the Penicillium chrysogenum ACV synthetase are essential for valine epimerization and processivity of tripeptide formation. Biochimie 2012, 94, 354-364. [CrossRef] [PubMed] 
114. Zompra, A.A.; Galanis, A.S.; Werbitzky, O.; Albericio, F. Manufacturing peptides as active pharmaceutical ingredients. Future Med. Chem. 2009, 1, 361-377. [CrossRef] [PubMed]

115. Zimmermann, G. Review on safety of the entomopathogenic fungi Beauveria bassiana and Beauveria brongniartii. Biocontrol Sci. Technol. 2007, 17, 553-596. [CrossRef]

(C) 2020 by the authors. Licensee MDPI, Basel, Switzerland. This article is an open access article distributed under the terms and conditions of the Creative Commons Attribution (CC BY) license (http://creativecommons.org/licenses/by/4.0/). 